\title{
Quantitative Feedback Control of Multiple Input Single Output Systems
}

\author{
Javier Rico-Azagra, ${ }^{1}$ Montserrat Gil-Martínez, ${ }^{1}$ and Jorge Elso ${ }^{2}$ \\ ${ }^{1}$ Electrical Engineering Department, University of La Rioja, 26004 Logroño, Spain \\ ${ }^{2}$ Automatic Control and Computer Science Department, Public University of Navarra, 31006 Pamplona, Spain \\ Correspondence should be addressed to Javier Rico-Azagra; javier.rico@unirioja.es
}

Received 24 September 2013; Revised 9 December 2013; Accepted 13 December 2013; Published 7April 2014

Academic Editor: Baoyong Zhang

Copyright (c) 2014 Javier Rico-Azagra et al. This is an open access article distributed under the Creative Commons Attribution License, which permits unrestricted use, distribution, and reproduction in any medium, provided the original work is properly cited.

This paper presents a robust feedback control solution for systems with multiple manipulated inputs and a single measurable output. A structure of parallel controllers achieves robust stability and robust disturbance rejection. Each controller uses the least possible amount of feedback at each frequency. The controller design is carried out in the Quantitative Feedback Theory framework. The method pursues a smart load sharing along the frequency spectrum, where each branch must either collaborate in the control task or be inhibited at each frequency. This reduces useless fatigue and saturation risk of actuators. Different examples illustrate the ability to deal with complex control problems that current MISO methodologies cannot solve. Main control challenges arise due to the uncertainty of plant and disturbance models and when a fast-slow hierarchy of plants cannot be uniquely established.

\section{Introduction}

This paper deals with systems where multiple inputs are used to govern a single output. Although scientific literature refers to them with diverse names, here they will simply be called MISO systems. In some cases, each individual output is accessible, as in distributed energy generation systems [1], decentralised production systems [2], or unmanned fleets [3]. Usually, this leads to complex multiloop control structures [4]. However, a lot of engineering MISO systems lack physical individual outputs or sensors to measure them. Such systems are common in process industry [5], where the MISO control sometimes pursues the management of the global production system [6], whereas other times it governs low-level process variables (typical examples are two pumps or a pump and a valve, used as actuators in pressure or flow control [7-9]). In other cases the MISO control attends certain subsystems in a process, such as chemical reactors [10-12] or biological reactors [13-15]. More specific usages can be found in drying sections of paper machines $[7,16]$ or in aerobic digesters of waste water treatment plants [17]. Heat exchangers [18$20]$, chemical reactors in polymerization processes $[9,18]$, or distillation columns $[9,20-23]$ are repeated references in the scientific literature as MISO control applications. The automotive industry has also adopted these principles, firstly for the government of internal combustion engines [24-27] and recently for HCCI (Homogeneous Charge Compression Ignition) engines [28-30]. Another area devoted to MISO control is the consumer electronics, and particularly the massive data storage devices [31-33]. And finally, biological engineering applications can be found in $[18,34]$.

Within those MISO systems with nonindividual measurable outputs, the control strategies can be divided into noncollaborative and collaborative ones. Noncollaborative control selects a plant inside a battery of them, which covers a wide range of operating points for the output. The selection criterion is based on the stationary capacity of each plant. Thus, the control law is designed for an equivalent SISO system. A selector splits online the control action to the plant or plants with capacity to regulate the output in the actual operating point. The split-range control $[35,36]$ is the most representative of this methodology. A simpler method reduces to a pure SISO control system, which closes a single feedback loop around a plant. The inputs to the other plants are manipulated manually or are left constant [37]. 
On the other hand, collaborative strategies benefit from the dynamic strengths of each plant to improve the controlled output performance considering the restrictions of manipulated variables and individual outputs. In the scientific literature collaborative MISO systems appear under diverse designations: VPC (Valve Position Control) [8, 9, 2123], habituating control [10-12, 17, 18, 34, 38], main-vernier control [39-42], cooperative-feedback control [20, 43, 44], load sharing control [2, 45-47], or PQ design method [27, 48, 49]. Midranging control $[7,13,15,16,35,50-54]$ is frequently found in process control literature.

The controlled collaboration of parallel plants can be performed by serial (Figure 1(a)) or parallel (Figure 1(b)) structures of controllers. Serial collaboration is based on a qualitative organization of plants: the main loop $c_{n} p_{n}$ corresponds to the fastest plant $p_{n}$, the first one to react to output deviations. The vernier loops are arranged to produce a chained intervention of gradually slower plants. The last loop, which incorporates the slowest plant, is in charge of the steady state. In this way, each system collaborates to the extent it provides benefits, avoiding useless actuator fatigue and risk of saturation. This is also the goal of parallel structures. However, the involvement of each plant is determined by its preceding controller, instead of by its position in the overall scheme. Thus, the design of each openloop transfer function $c_{m} c_{i} p_{i}$ becomes more complex, since it has to define both the actuation domain and the action itself. However, unlike serial structures, parallel designs can cope with systems where the fast-slow hierarchy cannot be uniquely established.

In addition to all this, a proper design must pay some attention to the pervasive presence of uncertainty. In this sense, some solutions for the MISO control problem come from the Quantitative Feedback Theory (QFT). Horowitz $[4,55]$ did not pay much attention to the MISO problem with no individual measurable outputs. His work suggests the reduction of the MISO system to a SISO equivalent by plant summation. Then, a master controller $\left(c_{m}\right.$ in Figure $1(\mathrm{~b})$ ) is designed for the equivalent plant, and no individual controller is used $\left(c_{i}=1, i=1, \ldots, n\right)$. The master control strategy can extract the maximum dynamic potential of each plant under certain restrictions for their phases. Nevertheless, its main drawback is that the same control action reaches all plant actuators. This entails using more feedback than necessary at certain frequencies on each branch, which leads to different negative effects in real-life actuators. In particular, an excess of noise amplification arises in the control actions to the slowest plants and steady-state offsets reduce the available actuation range for the fastest ones. Another drawback of plant summation strategy is that feedback is allocated by the plant instead of by the designer, losing some flexibility. This might have some importance in certain systems [7] whose high operation expenses suggest to arrange plant interventions according to monetary criteria rather than dynamic ones. Further QFT developments include the approaches in $[25,56]$, which detail a collaborative control of parallel plants with uncertainties and restrictions for particular applications, and the approach in [3], which adds feedback loops of actuations inside a parallel structure as described in [57].

However, a general robust methodology to deal with MISO collaborative systems through QFT tools has not been presented yet. Such is the goal of this paper. In particular, it focuses on the disturbance rejection or regulation problem. The new technique is fitted to the parallel structure. This is the most versatile one since other arrangements can always be transformed into it while the inverse transformation is not always feasible. Besides, some examples will show that mainvernier design philosophy cannot cope with certain types of MISO plants. The new methodology seeks a quantitative division of feedback amongst parallel loops in the frequency domain. In absence of restrictions, this distribution leads to the accomplishment of the specifications with minimum use of feedback, that is, each loop only contributes at those frequencies where its plant favours the output performance.

\section{MISO QFT Design Methodology}

2.1. Robust MISO Regulation Problem. Consider Figure 1(b) with $c_{m}=1$. Each parallel plant $p_{i}, i=1, \ldots, n$, defines the effect of each manipulated variable $u_{i}$ over the single measurable output $y$. The plant $p_{d}$ defines the way in which the nonmeasurable disturbance $d$ deviates the single output $y$ from its desired constant set-point $r$. A set of parallel controllers $c_{i}, i=1, \ldots, n$ are designed to minimize such deviation. Each $c_{i}$ fixes its corresponding $u_{i}$ based on a common output measurement $y$, which is corrupted by the noise signal $n$. For this regulation problem, the closed loop functions are

$$
\begin{gathered}
y=\frac{p_{d}}{1+l_{t}} d-\frac{l_{t}}{1+l_{t}} n, \\
y_{i}=-\frac{l_{i} p_{d}}{1+l_{t}} d-\frac{l_{i}}{1+l_{t}} n, \\
u_{i}=-\frac{c_{i} p_{d}}{1+l_{t}} d-\frac{c_{i}}{1+l_{t}} n,
\end{gathered}
$$

where $l_{i}=p_{i} c_{i}$ are the individual open-loop transfer functions and

$$
l_{t}=\sum_{i=1}^{n} l_{i}
$$

is the global open-loop transfer function.

All plants are assumed to present parametric uncertainty. Let us define $\mathbf{q}=\left[q_{1}, q_{2}, \ldots, q_{m}\right]$ as the vector of all parameters appearing in the transfer functions $p_{1}, p_{2}, \ldots, p_{n}, p_{d}$. Each $q_{i}$ varies within certain lower and upper limits. Therefore, the uncertainty vector $\mathbf{q}$ belongs to a hyperrectangle in $\mathbb{R}^{m}$ called the uncertainty space $Q$; that is,

$$
\mathbf{q} \in \mathbb{Q} \triangleq\left\{\mathbf{q} \in \mathbb{R}^{m} \mid q_{i}^{-} \leq q_{i} \leq q_{i}^{+}, i=1, \ldots, m\right\} .
$$

The purpose of QFT control is to enforce the specifications for all elements in the uncertainty space. In this case, the specifications include robust stability,

$$
\left|\frac{l_{t}}{1+l_{t}}\right| \leq W_{s} \quad \forall \mathbf{q} \in \mathbb{Q},
$$




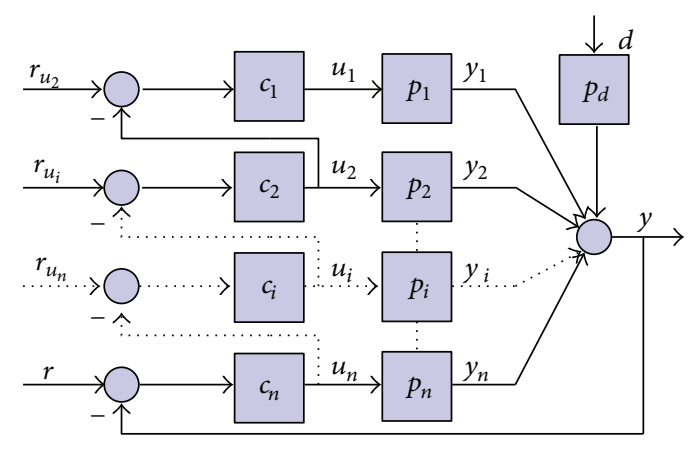

(a) Serial structure or main-vernier

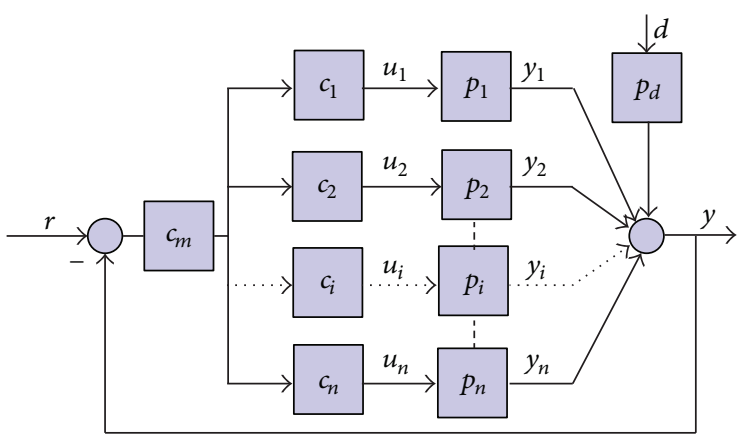

(b) Parallel structure

FIGURE 1: Control structures for MISO plant.

and robust disturbance rejection,

$$
\left|\frac{p_{d}}{1+l_{t}}\right| \leq W_{d} \quad \forall \mathbf{q} \in \mathbb{Q},
$$

where $W_{s}$ and $W_{d}$ are upper tolerances for each closed-loop frequency response.

As long as feedback control guarantees expected specifications at a certain price, QFT advocates for that control solution with the minimum amount of feedback, which will be denoted as the QFT optimum. In SISO control, the bounds express the minimal gain requirements on the nominal open-loop transfer function for the accomplishment of the specifications. Consequently, an QFT optimal loopshaping tries first a narrow fulfilment of the bounds, and then an abrupt roll-off to minimize the presence of noise in the control signals [58].

In the MISO case, the search for the QFT optimum is not so obvious. There is a minimal gain of the nominal open-loop global function $l_{t_{o}}$ that meets (4) and (5). However, it can be achieved by several combinations of $l_{i_{0}}$ in (2). That combination of minimum gains of each $c_{i}$ at each frequency is the solution that demands the minimum amount of feedback. Then, one option is splitting the global problem in several designs $l_{i_{o}}$ in such a way that each bound arrangement shows the minimum amount of feedback solution. If so, each loopshaping reduces to a narrow fulfilment of the bounds and a maximal reduction of gain in the frequencies where no restrictions are imposed.

In practice, the new methodology looks for a wise distribution of feedback, which exploits the power of each plant frequency response to achieve controllers of minimum gain. This minimizes the presence of noise in the control signals to the slowest plants, which usually exhibit the most powerful actuators and provide the bias action. In this way, faster plant actuators preserve their full range of operation for output regulation in response to disturbances.

2.2. Parallel Plants and the Amount of Feedback. The core of the method is to quantify the amount of feedback, which is evaluated with the controller gain. For a better understanding, let us take a single frequency, exclude the uncertainty, and consider solely the specification of robust disturbance rejection. Then, certain $l_{t}$ will be a solution to (5). Two parallel structures from Figure 1(b) will be studied: a master control, which sintonizes $c_{m}$, and does each $c_{i}=1$, and a strictly parallel control, which sintonizes each $c_{i}$ and does $c_{m}=1$.

To achieve $l_{t}$, we are firstly comparing the controller gain necessities for plants of similar characteristics. For simplification purposes, let us take two plants $p$. As expected, if the system power to regulate the output was increased from $p$ to $2 p$, feedback necessities would reduce in the same proportion from $\left|l_{t} / p\right|$ to $\left|l_{t} / 2 p\right|$, independently if a master controller or two parallel controllers were used.

Secondly, control necessities to achieve $l_{t}$ are being evaluated for plants with very different gains at the same phase. Let us take two plants as $\left|p_{1}\right| \gg\left|p_{2}\right|$. Note that, if $p_{1}$ plant worked alone, the feedback demand would be considerably inferior to the case of $p_{2}$ working alone: $\left|l_{t} / p_{1}\right| \ll\left|l_{t} / p_{2}\right|$. Therefore, there is no sense in using the $p_{2}$ actuation unless you consider other criteria different from meeting the performance with the minimum feedback. In the case of a master controller, its control demand is $\left|l_{t} /\left(p_{1}+p_{2}\right)\right|$, which approaches to $\left|l_{t} / p_{1}\right|$. Thus, $p_{1}$ is the only plant contributing to the performance and $p_{2}$ actuator is being excited unnecessarily. A parallel controller structure can overcome this drawback as follows. Basing on the formulation, $l_{t}=$ $c_{1} p_{1}+c_{2} p_{2}$ can be achieved by different combinations of $c_{1}, c_{2}$. However, as long as $p_{2}$ powerfulness is negligible, any attempt of collaboration with $p_{1}$ to the performance will require a huge control demand in the second branch. Then, the best option is to achieve the performance with the $p_{1}$ branch, whose control demand will be $\left|l_{t} / p_{1}\right|$. Simultaneously, tuning $c_{2} \approx 0$ avoids that useless signals reach the $p_{2}$ actuators.

One of the above two situations (plants that should collaborate or not) arises in the loop-shaping of controllers at each frequency. In a first stage of the proposed methodology, special QFT bounds guide the designer to define when several plants are similar enough to collaborate at certain frequency or when they are different enough such that some must take the whole responsibility and others must be inhibited. Model uncertainty and the whole set of specifications are included in the method. In a second stage, the feedback is 
shared accordingly through the sequential loop-shaping of controllers in the parallel structure.

\subsection{First Stage: Load Sharing Planning at Each Frequency of} Design. The proposed method firstly evaluates the amount of controller gain $\left|c_{i}\right|$ that each plant $p_{i}$ would demand to fulfil the specifications (4), and (5) by itself, that is, assuming $c_{j \neq i}=$ 0 . The required gain is expressed graphically by a QFT bound at each frequency $\omega$, denoted by $\beta_{c_{i}}(\omega)$. Note that, unlike common QFT bounds, these are bounds on the controller gain, not on the nominal open-loop gain $\left|l_{i_{0}}\right|$, in the vertical axis. This is so, because their purpose is not to serve as design guidelines, but to allow a graphical comparison among the controller gains that are being demanded at subsequent design phase $\angle l_{i_{o}}$ by each plant. Once all the bounds for a single frequency $\omega$ are computed and a design phase $\Theta_{d}$ is selected for comparisons, a conclusion is reached according to the following criteria.

(a) If one of the bounds, say $\beta_{c_{k}}(\omega)$, is sensibly lower than the others at $\Theta_{d}$, then regulation at that frequency must be accomplished through the openloop function $l_{k}$, and the other branches must be inhibited by decreasing the gain of $c_{i \neq k}(j \omega)$ as much as possible. The quantity of $20 \log n \mathrm{~dB}$ is a practical guideline to establish the bound height difference. It is funded on the ideal collaboration of $n$ equal plants, which would reduce $n$ times the amount of feedback, as Section 2.2 details. Figure 2 shows the analysis for a 2-branch structure, which suggests that the controller $c_{2}$ should carry the burden of control at $\omega_{1}$ whatever the chosen $\Theta_{d}$ is.

(b) If several bounds are located at the bottom and their heights do not differ too much from each other at $\Theta_{d}$, then their associated controllers must share the regulating task. The branches whose bounds locate above this bound group must be inhibited. In this case, the practical rule of thumb is that any set of $m$ bounds within a range of $\pm 20 \log m \mathrm{~dB}$ are suitable for cooperation. According to this, Figure 2 suggests that controllers $c_{1}$ and $c_{2}$ should collaborate at $\omega_{2}$ whatever the chosen $\Theta_{d}$ is to fulfil the specification.

Remark 1. The first stage of the new methodology arranges the best load sharing amongst parallel plants at each frequency for minimum amount of feedback in each branch to meet the control specifications. This approach is completely new inside the MISO control literature.

\subsection{Second Stage: Feedback Sharing in Parallel Structure of} Controllers. Once it is decided which plants or plants must deal with the specifications at each frequency, the $n$ parallel controllers are designed sequentially. The procedure presented here aims to minimize the number of iterations required to reach a feasible solution. At each step, one of the controllers is tuned by QFT loop-shaping, while the remaining ones are considered constant components of the overall uncertain system. This rotating nature of the tunable controller is enabled by the genbnds command of the Terasoft QFT Toolbox [59], which admits general specifications in the form

$$
\left|\frac{A+B G}{C+D G}\right| \leq W .
$$

Thus, if at some point the controller $c_{k}$ is to be adjusted, the robust stability (4) bounds can be computed by choosing $A=\sum_{i \neq k} l_{i}, B=p_{k}, C=1+\sum_{i \neq k} l_{i}, D=p_{k}, G=c_{k}$, and $W=W_{s}$, while the robust disturbance rejection (5) bounds require the choice $A=p_{d}, B=0, C=1+\sum_{i \neq k} l_{i}$, $D=p_{k}, G=c_{k}$, and $W=W_{d}$. Note that these are common QFT bounds, since they are imposed on the openloop transfer function $l_{k_{o}}$, and consequently denoted $\beta_{l_{k}}(\omega)$, which represent all the specifications to meet at $\omega$.

The design procedure begins with all controllers set to zero. Subsequently, in the first step of design, the loopshaping of the branch/branches that should work at the lowest frequency is performed fully. In an increasing order of frequencies, the last step corresponds with the design of branch/branches working at the highest frequency. During any loop-shaping design, the course of action at certain frequency depends on the result of the load sharing planning stage. As common in QFT for each loop-shaping, the design begins satisfying the bounds in an increasing order of frequencies, and finally, a fast roll-off of the open-loop gain is shaped in the high frequency range. A novelty of MISO control is that the open-loop gain must also be cut or reduced in the frequencies where the branch should be noncollaborating. Following, there are some hints of guide for both goals of design: when the branch must achieve the performance at $\omega$ and when the branch must be inhibited at $\omega$ because other branches do the work or because $\omega$ is the roll-off frequency for the branch.

At the frequencies where the branch/branches must perform the regulation task, two different cases are detailed next. Figures 3 and 4 show examples of both cases. Superscripts in bound designation indicate the step in the sequential design.

(a) A single plant $p_{k}$ will be responsible for the regulating task at $\omega$ (see the two-branch example in Figure 3). Thus, $l_{k_{o}}$ is placed above the bound $\beta_{l_{k}}(\omega)$ (see $l_{1_{o}}$ and $\beta_{l_{1}}^{0}$ ). After this, the $k$ bound arrangement does not change (see $\beta_{l_{1}}^{\mathrm{I}}$ ). However, since the accomplishment of the specifications has just been secured by $c_{k}$, the bounds $\beta_{l_{i \neq k}}(\omega)$ delimit now closed forbidden regions, which will allow a reduction of the $l_{i_{o} \neq k_{o}}$ gains for feedback saving at $\omega$ when these designs are faced (compare $\beta_{l}^{\mathrm{I}}$ with $\beta_{l_{2}}^{0}$ ). Note also that, an excess of $l_{k_{o}}$ gain to meet $\beta_{l_{k}}$ would imply a major contraction of $\beta_{l_{i \neq k}}$ if this was necessary in the design of the other loops.

(b) A group of $m$ plants will share the control task at $\omega$ (see the four-branch example in Figure 4, where $m=3$ ). Their loop shaping is carried out jointly as follows. For convenience, let us denote the group $p_{k_{1}}, p_{k_{2}}, \ldots, p_{k_{m}}$. Let us assume as well that $c_{k_{1}}$ is tuned first. The purpose of the designer is to place 


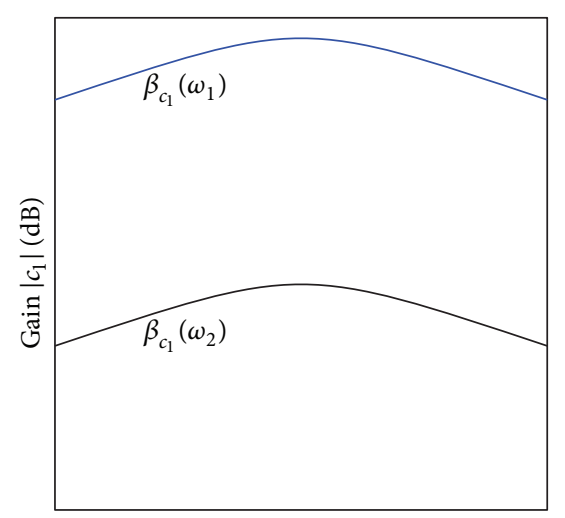

Phase $\angle l_{1}$ (deg)

(a)

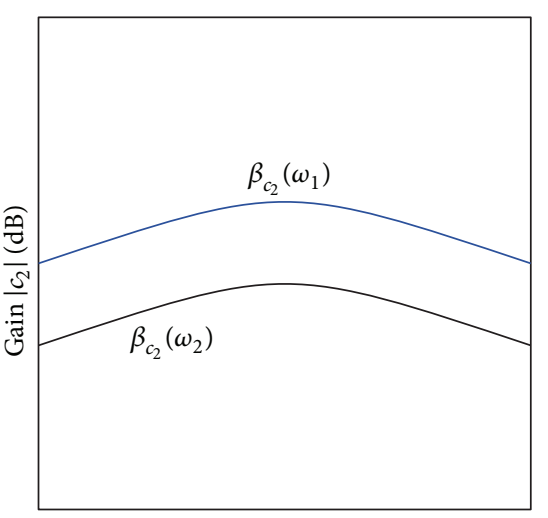

Phase $\angle l_{2}$ (deg)

(b)

Figure 2: Feedback demand comparisons in a $2 \times 1$ system.

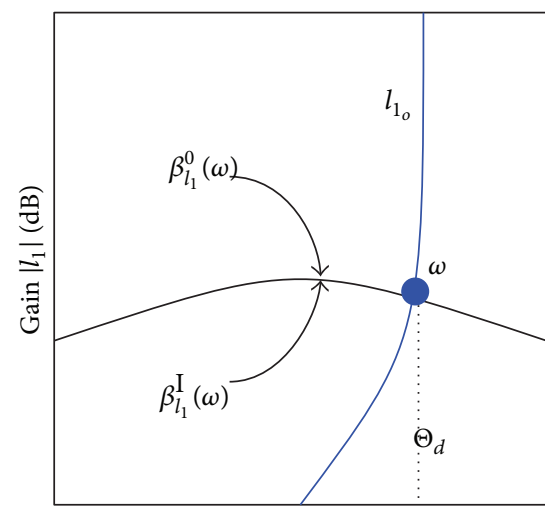

Phase $\angle l_{1}(\mathrm{deg})$

(a)

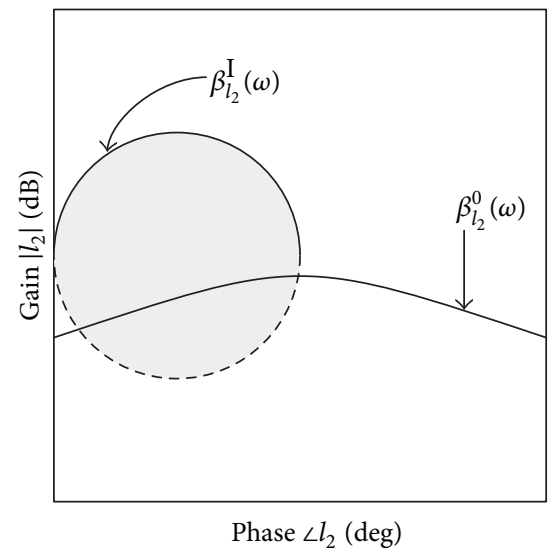

(b)

FIgURE 3: Single brach achieves the specifications at $\omega$ in a $2 \times 1$ system.

$l_{k_{1_{o}}}$ around $20 \log m$ decibels below the bound $\beta_{l_{k_{1}}}(\omega)$ at the design phase of interest $\Theta_{d}$ (see $l_{1_{o}}$ and $\beta_{l_{1}}^{0}$ ). The consequences are that $\beta_{l_{1}}(\omega)$ bound does not change (see $\beta_{l_{1}}^{\mathrm{I}}$ ) and that a dip appears at $\Theta_{d}$ in bounds $\beta_{l_{k_{j}}}(\omega), j=2, \ldots, m$ (see $\beta_{l_{2}}^{\mathrm{I}}$ and $\beta_{l_{3}}^{\mathrm{I}}$ ). Then, tuning of $l_{k_{2 o}}$ takes place, and the goal is to place $l_{k_{2 o}}$ around $20 \log (m-j+1), j=2$, decibels below the bound $\beta_{l_{k_{2}}}(\omega)$; (see $l_{2_{o}}$ and $\beta_{l_{2}}^{\mathrm{I}}$ ). Once achieved, the $\beta_{l_{k_{2}}}(\omega)$ bound does not change (see $\beta_{l}^{\text {II }}$ ), but it extends the depth of the dip in $\beta_{l_{k_{j}}}(\omega), j \neq 2$ (see $\beta_{l_{1}}^{\mathrm{II}}$ and $\beta_{l_{3}}^{\mathrm{II}}$ ). The process goes on in this manner, temporarily violating their bo-unds, until the tuning of $l_{k_{m_{o}}}$ is reached. In this case, the purpose is simply to meet the bound $\beta_{l_{k_{m}}}(\omega)$, whose dip has been repeatedly enlarged by each one of the controllers $c_{j}, j=1, \ldots, m-1$ (see $l_{3_{o}}$ and $\beta_{l_{3}}^{\mathrm{II}}$ ). Once this step is completed, all previously violated bounds are now met (see $\beta_{l_{1}}^{\text {III }}, \beta_{l_{2}}^{\text {III }}$, and $\beta_{l_{3}}^{\text {III }}$.
If the $m$ plants were identical at $\omega$ and $\Theta_{d}$, the gain of each controller $c_{k_{1, \ldots, m}}$ would be $20 \log m \mathrm{~dB}$ lower. Nevertheless, differences between plant gains up to $\pm 20 \log m \mathrm{~dB}$ are acceptable for successful cooperation and controller gain reduction, as Section 2.3(b) reports. In consequence, some extra adjustments are usually necessary to meet each $\beta_{l_{k_{j}}}$. After the design of the $m$ controllers with control responsibilities at $\omega$, the bounds of the remaining loops will delimit closed forbidden regions (see $\beta_{l_{4}}^{\text {III }}$ ).

At the frequencies where the branch/branches has/have no regulation responsibilities, a great deal of different situations may arise, specially when there are more than two inputs in the MISO system. Some of the most frequent ones are being described. In any case, the bound recalculation after any design step contributes to value the tradeoffs, which are inherent to any design with multiple degrees of freedom. 


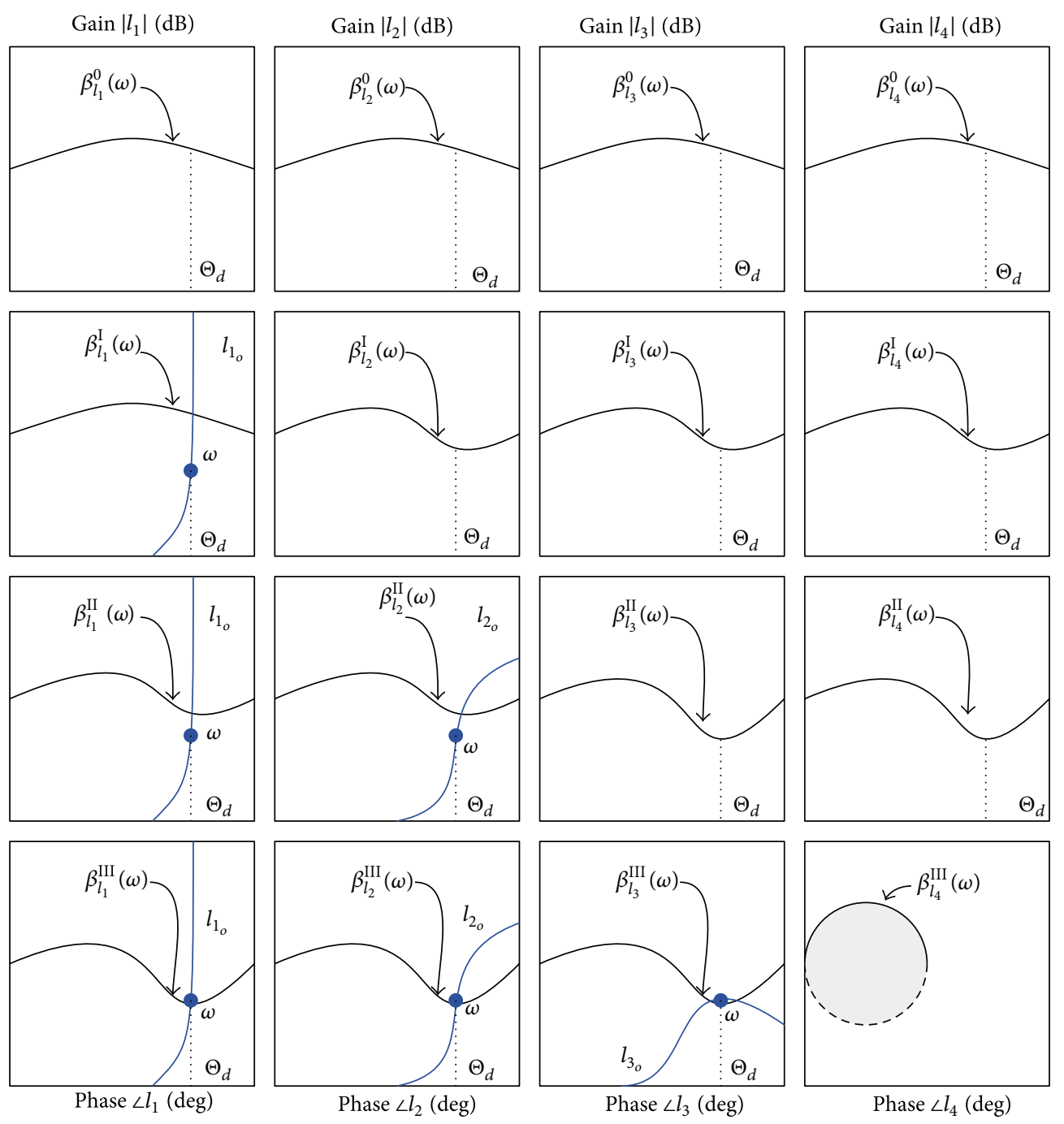

FIgURE 4: Three plants collaborate to achieve the specifications at $\omega$ in a $4 \times 1$ system.

(i) Design hints for the roll-off frequencies $\omega$ of the $k$ loop. The $l_{k_{o}}$ gain reduction is usually a tradeoff between a maximum noise amplification and a minimum controller order. One case is that the bounds $\beta_{l_{k}}$ demand certain feedback at $\omega$. This happens if there are other loops with control responsibilities at $\omega$ that have not been designed yet. In this circumstance, the $l_{k_{0}}$ design is performed temporarily violating its bounds at the roll-off frequencies $\omega$. After the design of the loops with control responsibilities at $\omega$, the bounds $\beta_{l_{k}}$ are automatically reshaped and now demarcate forbidden closed regions. In the new picture, $l_{k_{o}}$ will usually meet its bounds. Nevertheless, further iterations may be required to optimize the gain reduction at these roll-off frequencies. Other case is if the $k$ loop is the last one to be designed. In this circumstance, $\beta_{l_{k}}$ bounds, which already delimit forbidden closed regions, are the definitive restrictions to be met in the $l_{k_{o}}$ gain reduction at its roll-off frequencies. (ii) Design hints for the frequencies $\omega$ where the $k$ plant participation must be inhibited. The bounds $\beta_{l_{k}}$ currently demarcate fobidden closed regions at $\omega$, since other loops have already achieved the prescribed specifications. The restrictions for the $l_{k_{0}}$ gain reduction at frequencies $\omega$ are the bounds $\beta_{l_{k}}$ at frequencies higher than $\omega$, where the $k$ plant will have to achieve or collaborate in the regulation task. The controller complexity is another reason to take into account in the gain saving procedure.

Remark 2. The second stage of quantitative MISO control details the QFT framework and procedure for the sequential loopshaping of parallel controllers in order to meet the predefined load-sharing arrangement. A set of branches collaborate in the control tasks or are inhibited for feedback saving. A standard method is given to deal with $n$-input single-output systems from a quantitative feedback control point of view, which remained unsolved till now. 


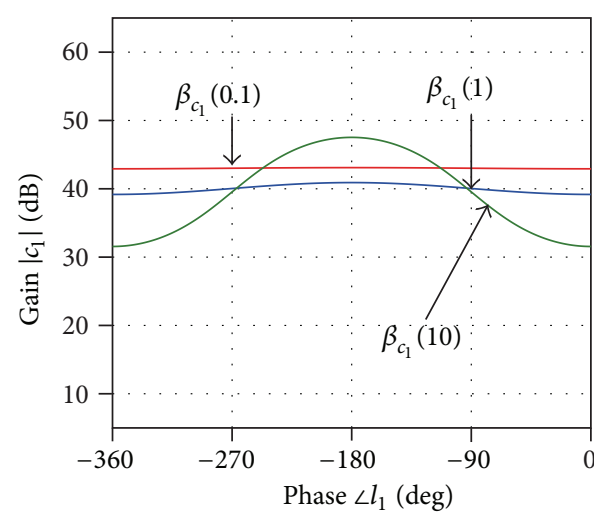

(a)

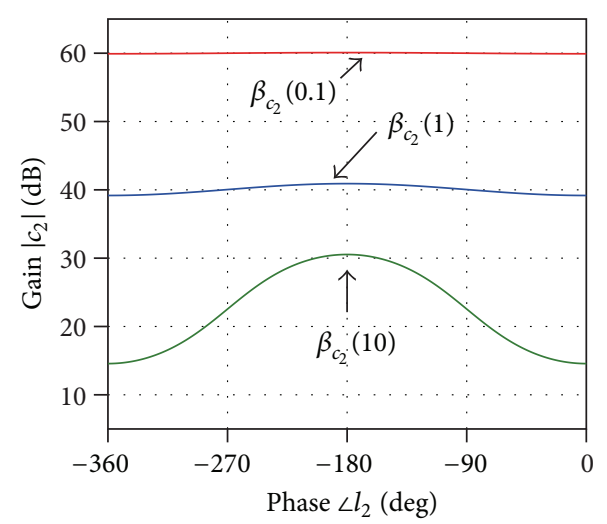

(b)

FIGURE 5: Feedback demands for single plant intervention.

\section{Design Examples}

3.1. Example 1. A simple MISO system illustrates the new methodology and its potential benefits. The uncertainty-free plant models are

$$
p_{1}=\frac{1}{10 s+1}, \quad p_{2}=\frac{0.1}{0.1 s+1}, \quad p_{d}=1 .
$$

Inside a parallel disposition (Figure 1(b) with $c_{m}=$ 1 ), the regulatory problem aims to design $c_{i=1,2}$ to meet certain stability (4) and performance (5) specifications with minimum controller gains at each frequency. For a minimum stability phase margin of $45^{\circ}$, the tolerance choice is

$$
W_{s}=\left|\frac{0.5}{\cos (\pi(180-P M) / 360)}\right|, \quad P M=45 .
$$

And for disturbance rejection, the performance upper model is

$$
W_{d}=\left|\frac{1.4167 s(s+30)}{(s+10)(s+42.5)}\right| \text {. }
$$

For bound computation and loop-shaping guide, a suitable vector of discrete frequencies is chosen:

$$
\Omega=\{0.1,0.5,1,5,10,100\}\left[\frac{\mathrm{rad}}{\mathrm{s}}\right] .
$$

3.1.1. First Stage: Load Sharing Planning at Each Frequency. According to explanations in Section 2.3, the required amount of feedback at $\Omega$ is computed in case that a single system regulated the output: $p_{1}$ or $p_{2}$, in Figure $5(\mathrm{a})$ and Figure 5(b), respectively (only three frequencies in $\Omega$ are drawn to illustrate the three potential outcomes). Compare controller bounds of both systems as follow.

(i) At low frequencies $(\omega<0.5)$, the bounds $\beta_{c_{1}}$ are over $20 \log 2 \mathrm{~dB}$ lower than the bounds $\beta_{c_{2}}$; see $\omega=0.1$. Thus, $p_{1}$ branch should assume $y$ regulation and $p_{2}$ branch should be switched off. Subsequent loop-shaping goals are $l_{t} \approx l_{1}, l_{2} \approx 0$ at $\omega<0.5$.
TABLE 1: Load-sharing planning for minimum amount of feedback.

\begin{tabular}{ccccccc}
\hline$\omega$ & 0.1 & 0.5 & 1 & 5 & 10 & 100 \\
\hline$p_{1}$ & $\times$ & $\times$ & $\times$ & & & \\
$p_{2}$ & & $\times$ & $\times$ & $\times$ & $\times$ & $\times$ \\
\hline
\end{tabular}

(ii) At medium frequencies $(0.5 \leq \omega<2)$, the bound heights differ less than $20 \log 2$; see $\omega=1$. Thus, the collaboration of both plants is suggested. Subsequent loop-shaping goals are $l_{1} \approx l_{2}$ at $0.5 \leq \omega<2$.

(iii) At high frequencies $\left(\omega \geq 2\right.$ ), the bounds $\beta_{c_{2}}$ are over $20 \log 2 \mathrm{~dB}$ lower than the bounds $\beta_{c_{1}}$; see $\omega=$ 10. Subsequent loop-shaping goals are $l_{t} \approx l_{2}, l_{1} \approx$ 0 at $\omega \geq 2$.

Accordingly, Table 1 summarises the load sharing planning at design frequencies $\Omega$.

3.1.2. Second Stage: Design of Parallel Controllers. Sequential loop-shaping of $c_{1}$ and $c_{2}$ is performed with Table 1 aims. Figure 6 illustrates the procedure in detailed steps. Steps 0 , I, and II belong to the same first iteration, which explains the design of $c_{1}$ and $c_{2}$ (marked with superscripts). Further iterations (step III) are required to optimize both designs looking for the strictly minimum amount of feedback at each frequency, which usually trades off with a reasonable order of the controllers. General guidelines were described in Section 2.4.

(0) Initial bound computation is drawn with $c_{2}^{0}=0$ for the loop 1 (Figure 6(a)) and with $c_{1}^{0}=0$ for the loop 2 (Figure 6(b)).

(I) Loop-shaping of $l_{1}$ (see Figure $6(\mathrm{c})$ ). To achieve $l_{t} \approx l_{1}$ at $\omega<0.5, l_{1}$ is located onto bound $\beta_{l_{1}}(0.1)$. For plant collaboration at $0.5 \leq \omega<2, l_{1}$ is shaped $20 \log 2 \mathrm{~dB}$ below $\beta_{l_{1}}(0.5)$ and $\beta_{l_{1}}(1)$. At $\omega \geq 2$, the goal becomes $l_{t} \approx l_{2}$. Then, $\omega \geq 2$ are the roll-off frequencies for $l_{1}$. Its gain is reduced as much as possible, even momentary violating bounds $\beta_{l_{1}}(5), \beta_{l_{1}}(10)$, and $\beta_{l_{1}}(100)$, 


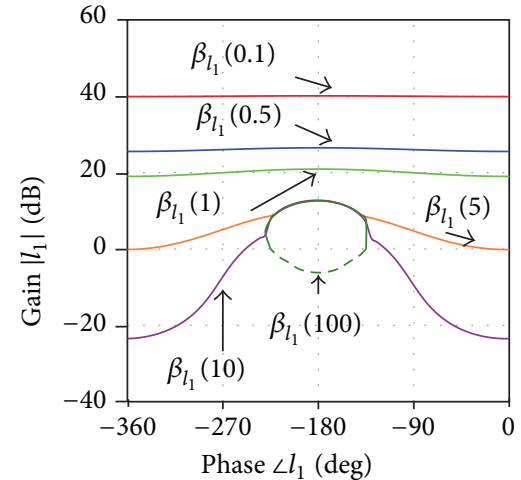

(a) Loop shaping $l_{1}^{\mathrm{o}}$

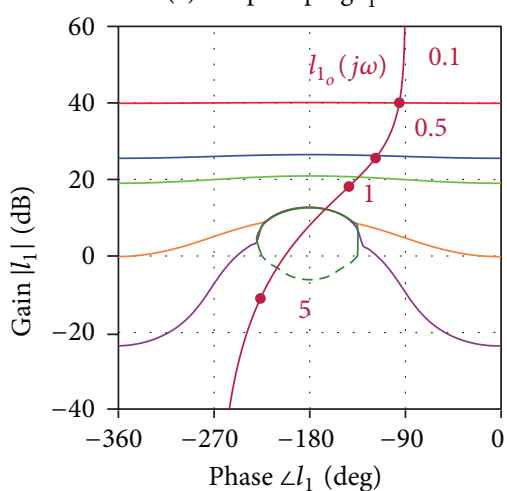

(c) Loop shaping $l_{1}^{\mathrm{I}}$

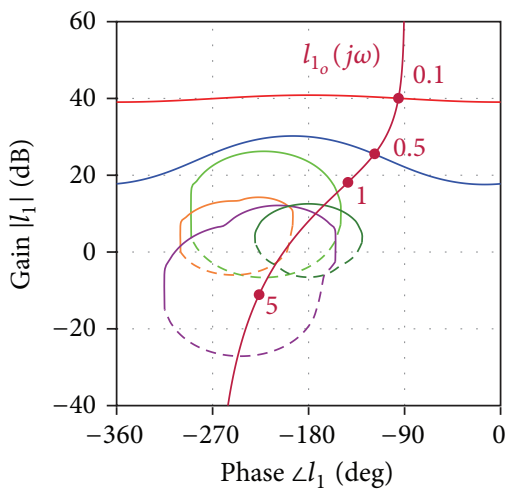

(e) Loop shaping $l_{1}^{\mathrm{II}}$

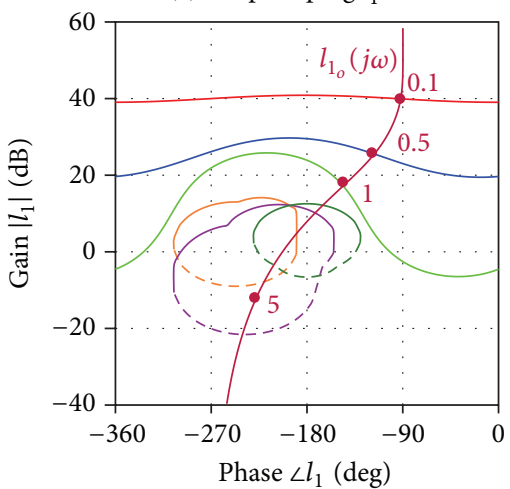

(g) Loop shaping $l_{1}^{\mathrm{III}}$

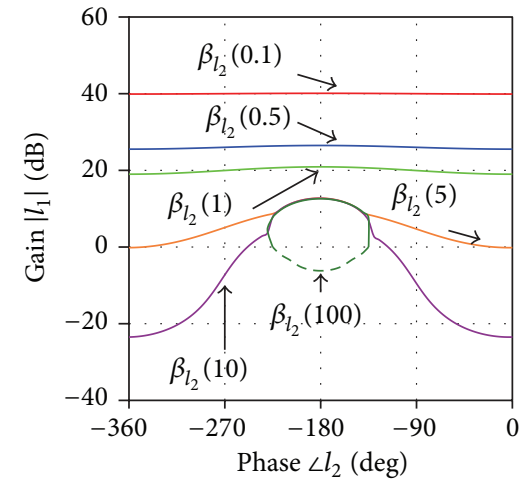

(b) Loop shaping $l_{2}^{\circ}$

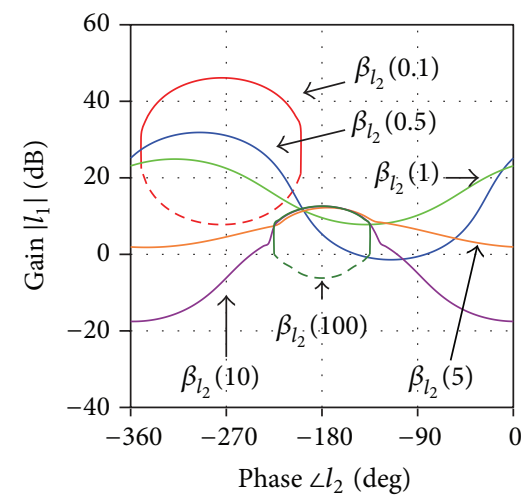

(d) Loop shaping $l_{2}^{\mathrm{I}}$

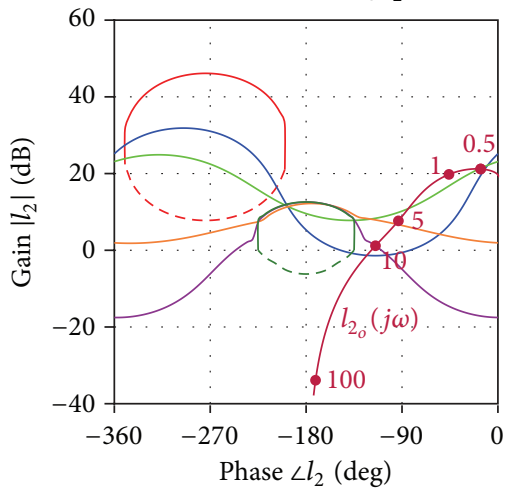

(f) Loop shaping $l_{2}^{I I}$

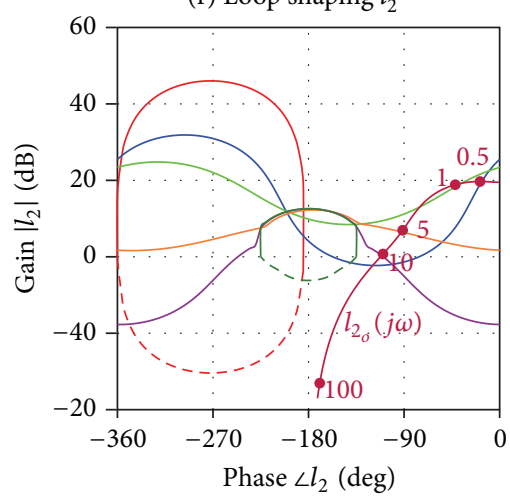

(h) Loop shaping $l_{2}^{\mathrm{III}}$

FIGURE 6: Sequential loop-shaping. 
since control requirements will be achieved by $l_{2}$. As this reduction is not bound guided, it could require later a $l_{1}$ reshaping at these high frequencies. After step I, the controllers are

$$
c_{1}^{\mathrm{I}}=\frac{417(s+0.1)}{s(s+2)^{2}}, \quad c_{2}^{\mathrm{I}}=c_{2}^{0}=0 .
$$

As a consequence, suitable bounds $\beta_{l_{2}}$ arise for the subsequent loop-shaping of $l_{2}$. Let us compare new bounds in Figure 6(d) with initial bounds in Figure 6(b): closed exclusion regions appear in $\beta_{l_{2}}(0.1)$ for $l_{2}$ feedback saving at $\omega<0.5$, since $l_{1}$ has already achieved the feedback requirements; dips arise in $\beta_{l_{2}}(0.5)$ and $\beta_{l_{2}}(1)$, where $l_{2}$ has to collaborate with previous $l_{1}$ contribution; the bounds at $\omega=$ $\{5,10,100\}$ are not modified since $l_{2}$ must assume the whole control tasks.

(II) Loop-shaping of $l_{2}$ (see Figure 6(f)). Firstly, reduce $l_{2}$ gain at $\omega<0.5$ to meet $\left|l_{2}\right| \ll\left|l_{m}\right|$, since $l_{t} \approx l_{1}$ was achieved. This example does not need adding a lag network, which favours a lower order of controller $c_{2}$. Secondly, locate $l_{2}$ onto its bounds $\beta_{l_{2}}$ at $\omega=\{0.5,1,5,10\}$; note that the purpose of an excess of feedback at $\omega=1$ is the illustration of subsequent optimization iterations. Finally, reduce the high frequency gain of $l_{2}$ at $\omega \geq 100$ (roll-off frequencies) as much as possible as in any classical QFT design. After step II, the controllers are

$c_{2}^{\mathrm{II}}=\frac{193.2(s+0.4)(s+6.3)}{(s+11)\left(s^{2}+1.2 s+0.51\right)}, \quad c_{1}^{\mathrm{II}}=c_{1}^{\mathrm{I}}$.

As a consequence, let us compare new bounds $\beta_{l_{1}}$ in Figure 6(e) with bounds of the previous step in Figure 6(c). Note that $l_{1}$ now meets all its bounds. Pay special attention to collaboration frequencies in Figures 6(e) and 6(f): the feedback sharing is close to the optimum at $\omega=0.5\left(l_{1}\right.$ and $l_{2}$ lie on their respective bounds), but it is not the case at $\omega=1$. Thus, small adjustments are required to economize feedback.

(III) Both loops are iteratively redesigned in Figures 6(g) and $6(\mathrm{~h})$. Thanks to the help of software tools, this is usually a simple procedure. The final controllers are

$$
\begin{gathered}
c_{1}=c_{1}^{\mathrm{III}}=\frac{329.7(s+0.088)}{s(s+1.5)(s+2)}, \\
c_{2}=c_{2}^{\mathrm{III}}=\frac{225.13(s+1.1)(s+6.95)}{(s+14.81)\left(s^{2}+1.77 s+1.22\right)} .
\end{gathered}
$$

Note that $l_{1}$ achieves by itself the control requirements at $\omega=0.1$ and shares this task with $l_{2}$ at $\omega=$ $\{0.5,1\}$. Thus, $l_{1}$ lies on $\beta_{l_{1}}$ at $\omega=\{0.1,0.5,1\}$. Alongside, $l_{2}$ achieves by itself the performance load at $\omega=\{5,10\}$. Thus, $l_{2}$ lies on $\beta_{l_{2}}$ at $\omega=$ $\{0.5,1,5,10\}$. It's also necessary to remark how the bound disposition eases the reduction of the openloop gains, in particular at $\omega>1$ for $l_{1}$ and at $\omega<0.5$, $\omega>10$ for $l_{2}$. Nevertheless, the feedback saving at these frequencies trades off with a minimum order of controllers.

3.1.3. Analysis and Comparisons. Parallel control, $c_{i=1,2}$ (13) and (14), is being compared with master control, $c_{m}$, which is designed for the equivalent plant $p_{e}=p_{1}+p_{2}$ in order to satisfy the same performance and stability specifications. QFT design yields the single controller

$$
c_{m}=\frac{192.6(s+0.1)(s+4.4)}{s(s+1)(s+8.9)} .
$$

For a fairer comparison, $l_{m}=c_{m} p_{e}$ lies exactly on its bounds and reduces its high frequency gain as much as possible. Thus, it is the solution of minimum amount of feedback at each frequency if the system was single input; Figure 7 (a) depicts the minimum $\left|c_{m}\right|$. This QFT optimal loop-shaping is easily achievable as there is no uncertainty and there is a single controller. For the MISO solution, the exact meeting of bounds with controllers (13) and (14) required a second iteration to reshape the former controllers (11) and (12). Nevertheless, the main feedback saving is not so much to lie exactly on the bounds but to share conveniently the feedback between branches. Note that $\left|c_{2}\right| \ll$ $\left|c_{m}\right|$ at low frequencies, where $p_{1}$ regulates the output, and $\left|c_{1}\right| \ll\left|c_{m}\right|$ at high frequencies, where $p_{2}$ works. In this way, $c_{2}$ filters low frequencies, which avoids unnecessary offsets of $p_{2}$ actuation. And $c_{1}$ filters high frequencies, which reduces the amplification of high frequency signals (sensor noise) in $p_{1}$ actuator (usually labelled as cost of feedback in SISO QFT [58]).

Figure 8 clearly demonstrates these benefits in the time domain. External inputs are a unity step disturbance $d(t)$ at $t=0.5 \mathrm{~s}$ and the sensor noise $n(t)$ built with a whitenoise source (noise power $=0.001$, sample time $=0.01$ ). All response signals are depicted with and without noise intervention in blue and green lines, respectively. As long as $l_{1}+l_{2} \approx l_{m}$ (a better agreement could be achieved with higher order of controllers), the closed-loop transfer functions $y / n$ and $y / d$ are similar for both control strategies. Accordingly, the $y(t)$ performance coincides for master and parallel control (only one is depicted in Figure 8), in presence (blue line) or absence (green line) of noise. However, the control effort strikes the differences as setting forward by closed-loop frequency responses in Figure 7(b). Let us compare the pair $u_{1}(t)-u_{2}(t)$ with the signal $u_{m}(t)$ in Figure 8 . Actuation $u_{1}(t)$ is hardly affected by sensor noise amplifications, which avoids unnecessary fatigue of $p_{1}$ actuator; the exact quantification of this benefit is on the distance 


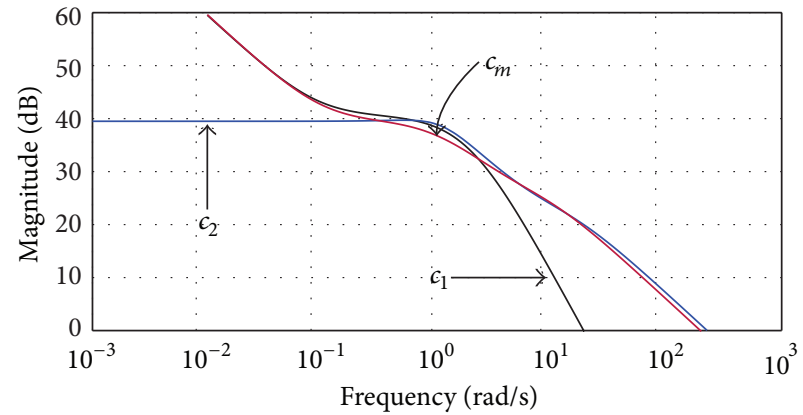

(a)

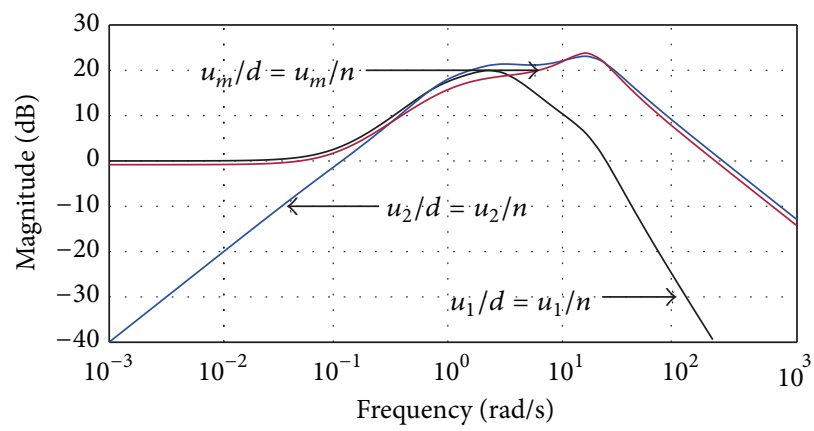

(b)

Figure 7: (a) Amount of feedback. (b) Closed-loop feedback cost.
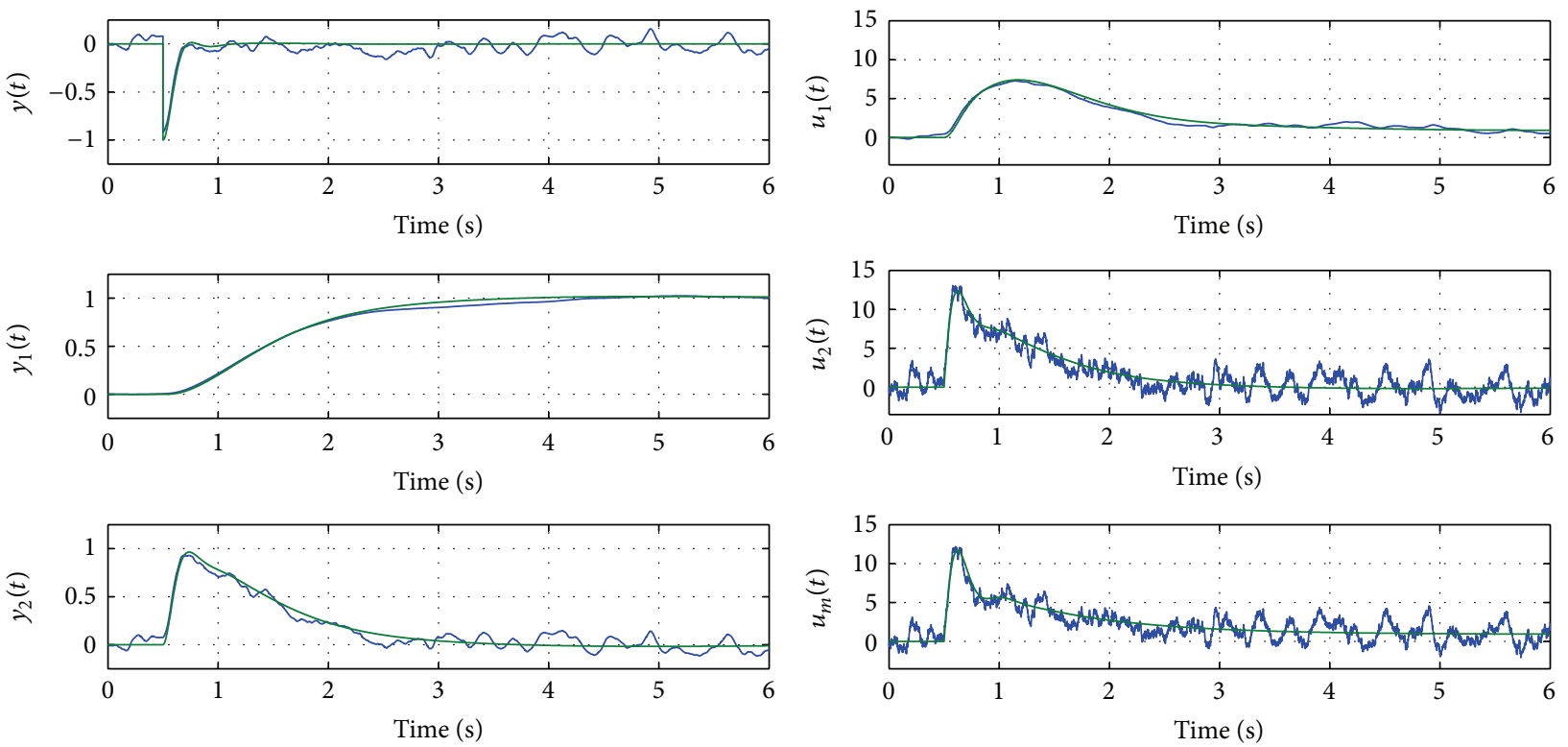

Figure 8: Time domain behaviour.

between $\left|u_{1} / n\right|$ and $\left|u_{m} / n\right|$, which mainly depends on the affordable bandwidth saving during the $l_{1}$ shaping. On the other hand, the noise $n(t)$ affects similarly to both $u_{2}(t)$ and $u_{m}(t)$. However, $u_{2}(t)$ recovers the initial stationary state and $u_{m}(t)$ does not: see $u_{2}(t=\infty) \approx 0, u_{m}(t=\infty)=u_{1}(t=$ $\infty)$ in time-domain, and $\left|u_{2} / d\right|_{\omega=0} \approx-\infty,\left|u_{m} / d\right|_{\omega=0} \approx$ $\left|u_{1} / d\right|_{\omega=0}$ in the frequency domain. Thus, for accumulative disturbances, the fast time actuation of $u_{2}(t)$ starts out always from zero, and consequently, the full range of $u_{2}$ signal is available to speed up the transient response with a less saturation risk. Both improvements (the noise release of $u_{1}(t)$ and the zero steady state of $\left.u_{2}(t)\right)$ are more valuable in practice, where real-life plants, actuators, and sensors have physical limitations.

As some final comparisons, once $c_{1}$ and $c_{2}$ are achieved, there are equivalences in other MISO control topologies (Eitelberg's master-slaves [46] and Lurie's serial structure [39]) for this particular example. Designs according to those other structures and methodologies can always be transformed into the strictly parallel structure, while the inverse transformation is not always feasible. In any case, the same QFT optimal solutions (13) and (14) would not probably be attainable since those other design methodologies do not quantify neither the amount of feedback nor the collaboration degree. Besides, some MISO plants or design-condition arrangements are only affordable with the new parallel design methodology; as following examples are discussing.

Remark 3. Firstly, this example details the two stages of the proposed MISO control methodology. Then analysis and comparisons show the expected benefits of a parallel structure of controllers in comparison with a single master controller: the reduction of useless fatigue and saturation risk of actuators. In comparison with other MISO control strategies (master-slaves or serial structures), the new one offers the minimum amount of feedback to govern each plant for prescribed specifications.

3.2. Example 2. Heat exchangers are common engineering applications of MISO control [18-20]. Figure 9 outlines 


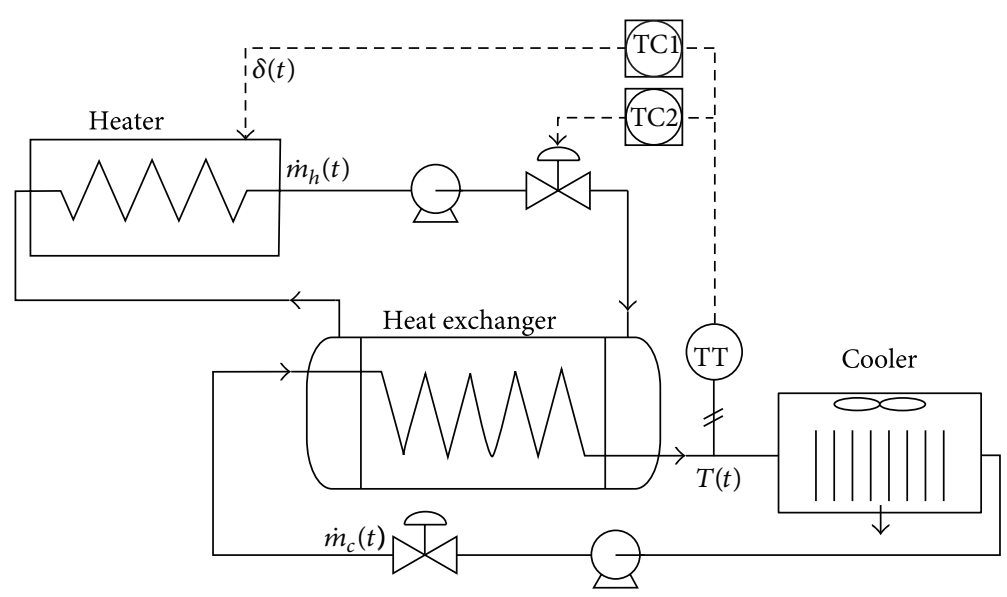

FIGURE 9: Thermal process control.

a heat-exchanger where the output temperature $T(t)$, can be regulated through two manipulated variables: the hot flow $\dot{m}_{h}(t)$ and the heating power control $\delta(t)$ (which conditions the input temperature of the hot flow). The cold flow $\dot{m}_{c}(t)$ acts as a disturbance since the production rate changes on demand. A laboratory-scale plant commercialised by Feedback Systems Inc. agrees with this arrangement [60].

Around certain operating conditions, linear plant models of the form

$$
p(s)=\frac{b_{3} s^{3}+b_{2} s^{2}+b_{1} s+b_{0}}{s^{4}+a_{3} s^{3}+a_{2} s^{2}+a_{1} s+a_{0}}
$$

are identified [61]. They express the relationships from the two manipulated variables to the output $\left(p_{1}=T / \delta\right.$ and $p_{2}=$ $\left.T / \dot{m}_{h}\right)$ and from the disturbance to the output $\left(p_{d}=\right.$ $\left.T / \dot{m}_{c}\right)$. A total amount of 112 cases are defined for each output/input relation, which generate the uncertainty of numerator and denominator coefficients in (16). Figure 10 depicts the envelopes of the plant frequency responses. As long as all the $p$-models are minimum phase, only magnitude Bode diagrams are included.

The robust stability control specification is (4) with tolerance (8) for a minimum phase margin of $45^{\circ}$. The specification for robust disturbance rejection is (5) with upper tolerance model

$$
W_{d}=\left|\frac{1.2 s(s+1.6)(s+5)}{(s+6)\left(s^{2}+1.6 s+1.778\right)}\right| .
$$

After computing the demand of feedback if $p_{1}$ or $p_{2}$ worked alone, Table 2 resumes the load-sharing planning at discrete frequencies to meet the robust control specifications with the minimum amount of feedback.

Consequently, the loop-shaping of $l_{1_{o}}$ and $l_{2_{o}}$ are performed (see Figure 11) and yield

$$
\begin{gathered}
c_{1}=\frac{69.5(s+0.0044)(s+0.058)}{s(s+0.018)(s+0.49)(s+2.94)}, \\
c_{2}=\frac{278.2(s+0.003)(s+0.052)(s+0.54)}{(s+0.0027)(s+0.016)(s+4)(s+20)} .
\end{gathered}
$$

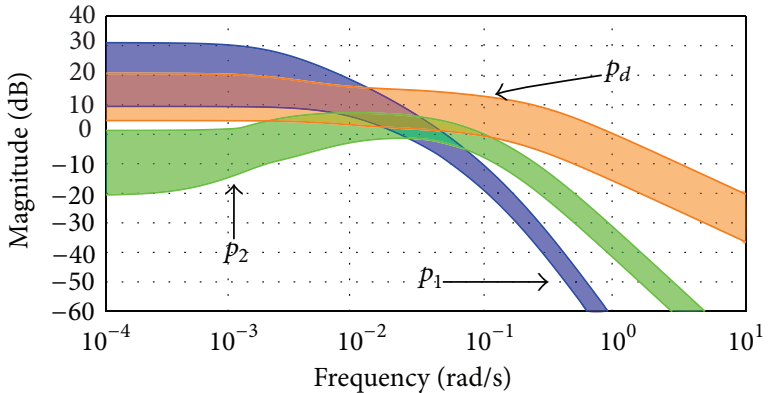

FIGURE 10: Frequency response magnitude plots of plants.

TABLE 2: Load-sharing planning along the frequency band.

\begin{tabular}{cccccccc}
\hline$\Omega$ & 0.005 & 0.02 & 0.05 & 0.2 & 0.5 & 1 & 10 \\
\hline$p_{1}$ & $\times$ & $\times$ & & & & & \\
$p_{2}$ & & $\times$ & $\times$ & $\times$ & $\times$ & $\times$ & $\times$ \\
\hline
\end{tabular}

In Figure 12, closed-loop frequency responses illustrate the achievement of control specifications and the control effort that is used for it. Only the envelopes of a set of 112 frequency responses are depicted for each plant. In summary, the originality of this example relapses in the engineering character of the MISO plant, the robustness of the new methodology (model uncertainty consideration), and the challenge of disturbance incorporation through a dynamical model with uncertainty.

For comparison purposes, a master controller is firstly designed for the summation of plants $p_{e}=p_{1}+p_{2}$ in order to meet the same robust control specifications

$$
c_{m}=\frac{280(s+0.0044)(s+0.058)(s+0.38)(s+0.58)}{s(s+0.018)(s+0.49)(s+4)(s+20)} .
$$

The parallel arrangement and the master controller behave equally attending to the prescribed stability and performance. However, the parallel one is superior in terms 


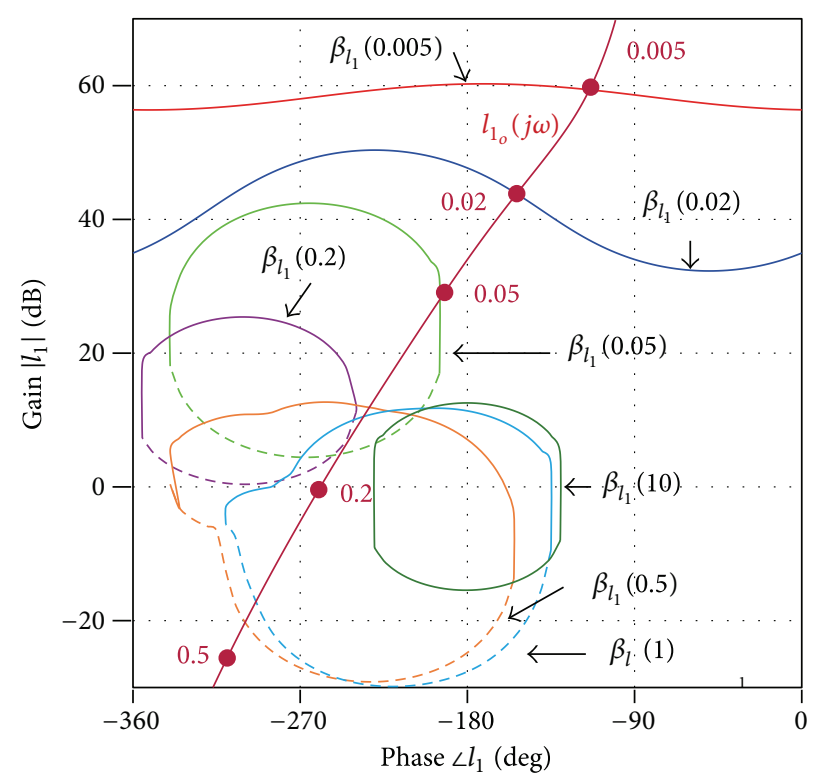

(a)

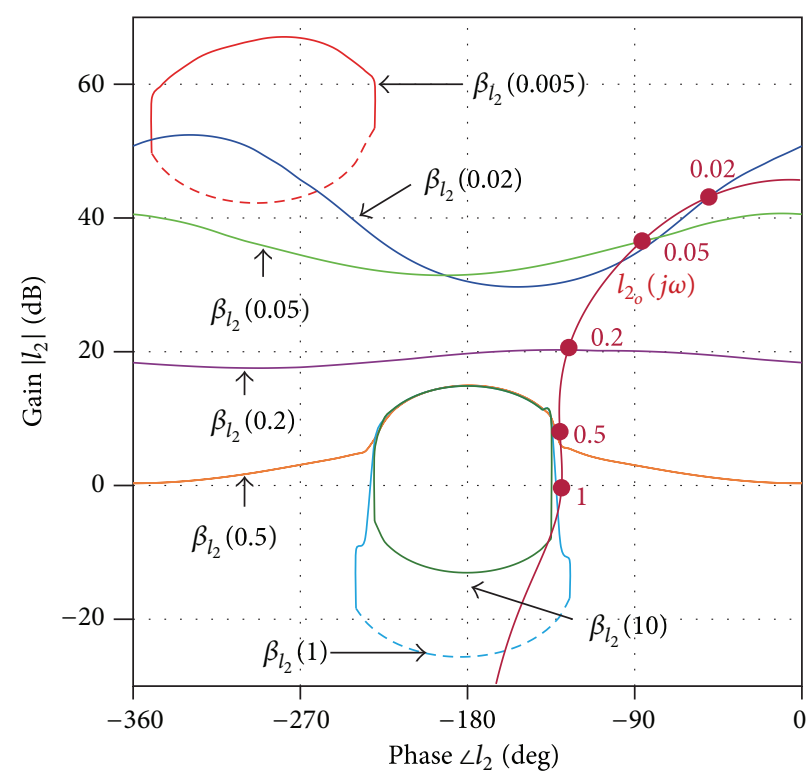

(b)

FIGURE 11: Loop-shaping of controllers.

of the actuation signals. The same reasoning and benefits of example 1 can be extended to this example.

Secondly, comparisons of the parallel arrangement and the master-slaves structures are shown. The master-slaves structure (Figure $1(\mathrm{~b})$ with $c_{m} \neq 1, c_{1} \neq 1$, and $c_{2} \neq 1$ ) can be considered a particular case of the parallel structure $\left(c_{m}=\right.$ $1)$, since this last one will be demonstrated more versatile. Attending their functionality as filters, let us rename slave $c_{1}$ as filter $f_{1}$ and slave $c_{2}$ as filter $f_{2}$. Let us suppose that $c_{1}$ (18) and $c_{2}$ (19) have already been designed for a parallel control structure. Then, there would not be a single equivalence for them in the master-slaves structure. Equivalents should meet: $c_{m} f_{1}=c_{1}$ and $c_{m} f_{2}=c_{2}$. A practical solution would be firstly to assume the master $c_{m}$ of (20), as long as its output performance matches that of the set $c_{1}$ (18) and $c_{2}$ (19). And secondly, to overcome the drawbacks of master control for parallel plants, filters are computed as $f_{1}=$ $c_{1} / c_{m}$ and $f_{1}=c_{1} / c_{m}$ with $c_{1}$ (18) and $c_{2}$ (19). This equivalence procedure is also reliable in example 1 . The inverse procedure, that is, the direct design of $f_{1}, f_{2}$, and $c_{m}$ can be successfully done in example 1; however, it would become extremely difficult in example 2 for the following reasons. The master-slaves method firstly addresses the design of the filters and then the master. As a first difficulty, the filters have to perform the load sharing in frequency terms, but their manual open-loop design would not meet quantitative closed-loop criteria. Besides, the uncertainty in parallel plant models $\left(p_{1}, p_{2}\right)$ hinders the choice of the cut-off frequency and the roll-off of the filters. Another difficulty is that the disturbance participation through dynamical models $\left(p_{d}\right)$ makes impossible to guess the best load sharing along the frequencies between the parallel plants $\left(p_{1}, p_{2}\right)$, and then it hinders a suitable parametrization of filters. At a second step of the master-slave method of design, the master would be designed, taking into account the initial MISO plant and the branch filters. In this stage, filter roll-offs may add serious problems: a high roll-off improves the filtering power but the branch phase deviates far from the original plant phase. Thus, branches can reach counter-phase conditions at certain frequencies. In such a case, a master controller spoils plant collaborations, since the vectorial sum $l_{t}=l_{1}+l_{2}$ yields a smaller modulus than $l_{1}$ and $l_{2}$.

Remark 4. A common engineering problem is solved: the robust regulation of output-flow temperature in a heat exchanger manipulating the input-flow heating power and the input heating flow itself, for different production rates (output-flows). Model uncertainty is fully considered to obtain the minimum amount of feedback controllers, whose benefits are detailed in previous examples. Additionally, the difficulties exhibited by the master-slaves strategy are overcome. The challenge is due to plant uncertainty and to the dynamical incorporation of disturbances.

3.3. Example 3. Despite the large number of engineering systems that have more than two manipulated inputs and single regulated output, few applications of this kind have been solved [40] so far. In fact, only a few scientific works incorporate a control theory for $n$ inputs $[25,26,45,48,56]$ because of its complexity. In this sense, the following example tries to demonstrate the potential of the new methodology. Consider a system with three manipulated inputs and a single regulated output, whose transfer function models are

$$
p_{i}=\frac{k_{i}\left(a_{i} s+1\right)}{\left(b_{i} s+1\right)\left(c_{i} s+1\right)}, \quad i=1,2,3
$$




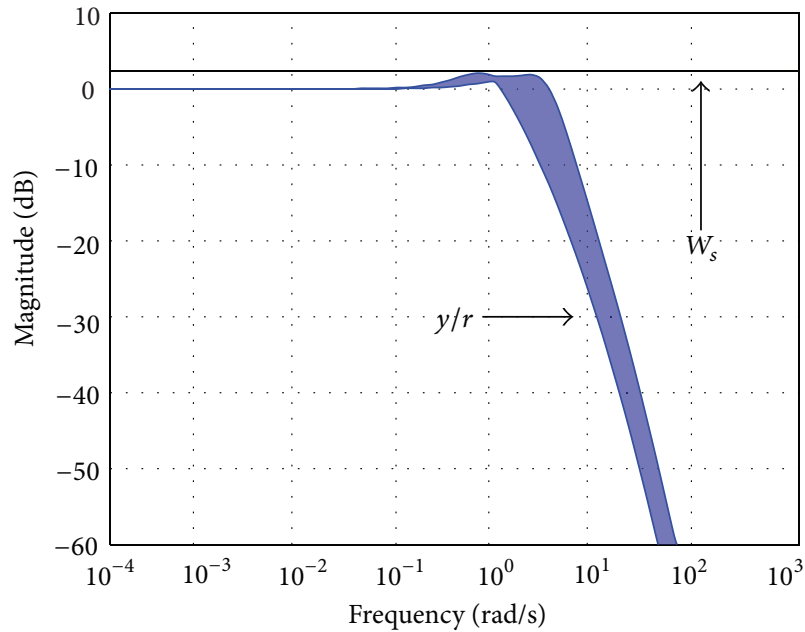

(a)

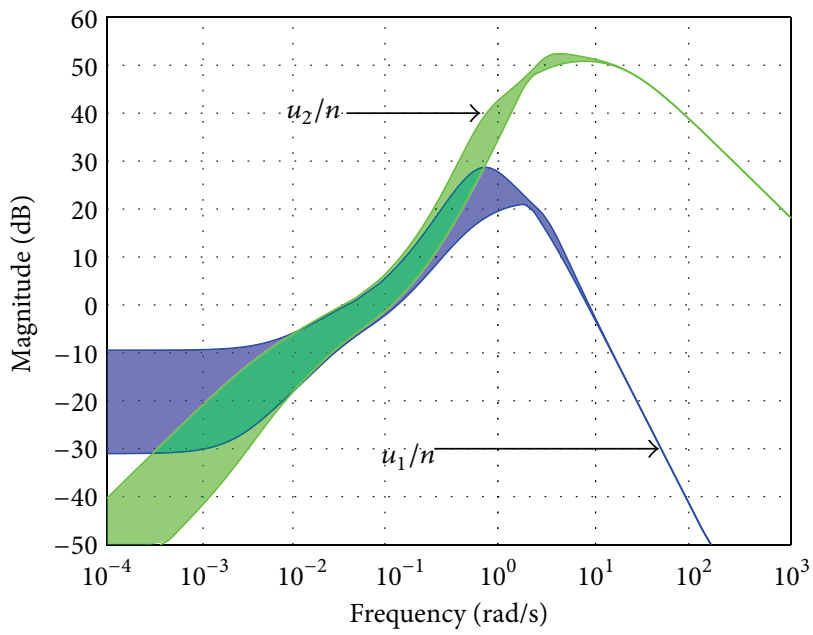

(c)

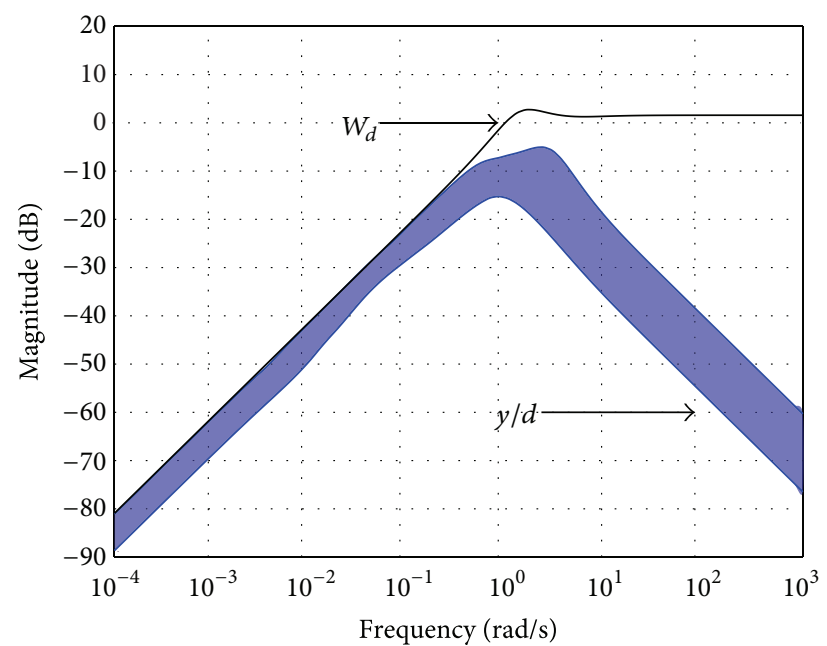

(b)

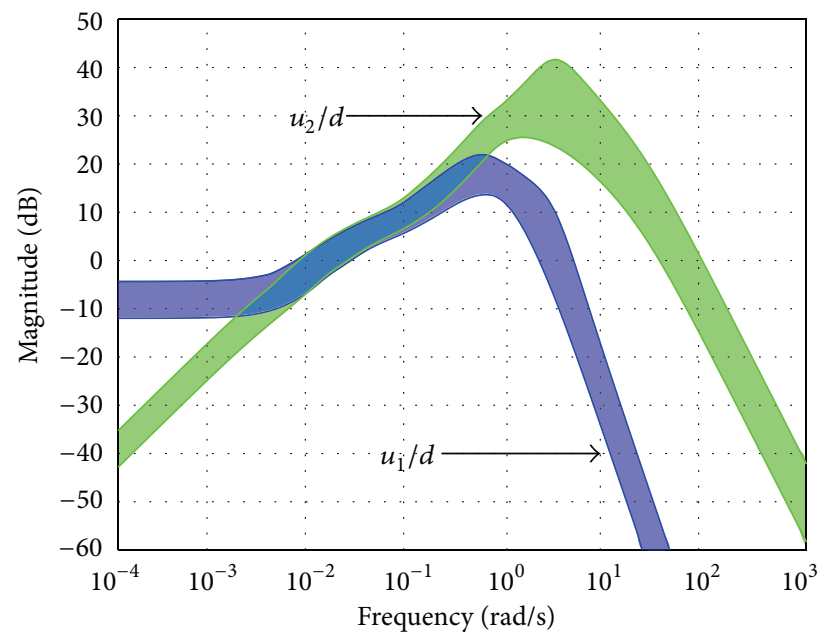

(d)

FIGURE 12: Closed-loop frequency response magnitude plots.

which are particularised for each $i$ input by $k_{1}=100, k_{2}=$ $10, k_{3}=1, a_{1}=10, a_{2}=a_{3}=0, b_{1}=1000, b_{2}=b_{3}=$ 0.01 , and $c_{1}=0.1, c_{2}=c_{3}=0$. Disturbances come into the single output straightforward, $p_{d}=1$. As the difficulty of this example relapses on dealing with three actuators, parameter uncertainty has been intentionally omitted in the $p_{i}$ plant models. Stability and disturbance rejection have to be attended meeting (4), (8) and (5), (9), respectively.

Upper section of Table 3 shows the amount of feedback $\left|c_{i}\right|$ that a single branch $c_{i} p_{i}$ would demand at the design phase $\Theta_{d} \approx-90^{\circ}$ to meet the specifications by itself. Note that the selection of design phase is part of the designer tasks. Previous examples depicted control necessities on the Nichols chart, that is, for all possible design phases $\left[-360^{\circ}, 0^{\circ}\right]$. Consequently with the upper part, the bottom of Table 3 shows the planning on load sharing along the frequencies; revise the criteria in Section 2.3 to decide plant collaborations or not as a function of feedback demand $\left|c_{i}\right|$.

Accordingly to results and choices on Table 3, the loop-shaping of each $l_{i}, i=1,2,3$ is sequentially performed
TABLe 3: (Up) Feedback demand; (down) load-sharing planning.

\begin{tabular}{cccccccccc}
\hline$\Omega$ & 0.001 & 0.01 & 0.05 & 0.1 & 0.5 & 1 & 5 & 10 & 100 \\
\hline$\left|c_{1}\right|$ & 43 & 40 & 39 & 37 & 26 & 20 & 6 & -3 & 20 \\
$\left|c_{2}\right|$ & 60 & 40 & 27 & 23 & 20 & 20 & 19 & 13 & 40 \\
$\left|c_{3}\right|$ & 80 & 60 & 46 & 40 & 26 & 20 & 5 & -6 & 3 \\
\hline$\Omega$ & 0.001 & 0.01 & 0.05 & 0.1 & 0.5 & 1 & 5 & 10 & 100 \\
\hline$p_{1}$ & $\times$ & $\times$ & & & $\times$ & $\times$ & $\times$ & $\times$ & \\
$p_{2}$ & & $\times$ & $\times$ & $\times$ & $\times$ & $\times$ & & & \\
$p_{3}$ & & & & & $\times$ & $\times$ & $\times$ & $\times$ & $\times$ \\
\hline
\end{tabular}

(see the final arrangements in Figure 13). The achieved controllers are

$c_{1}$

$=\frac{584.7(s+0.0007)(s+0.39)(s+7.68)\left(s^{2}+0.132 s+0.009\right)}{s(s+0.0068)(s+0.08)(s+9)(s+86)\left(s^{2}+0.98 s+0.33\right)}$, 


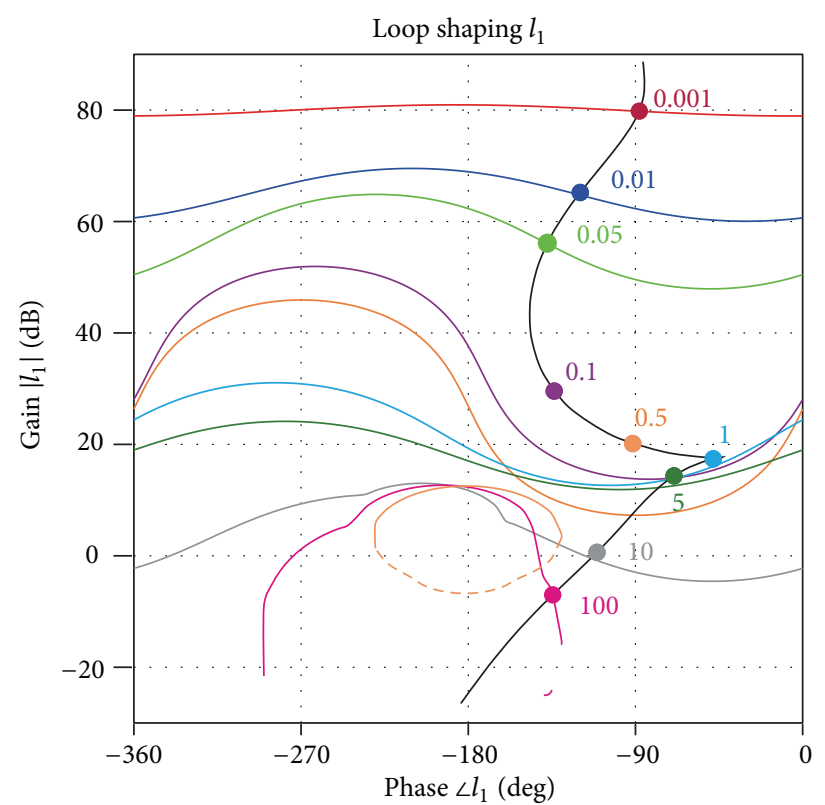

(a)

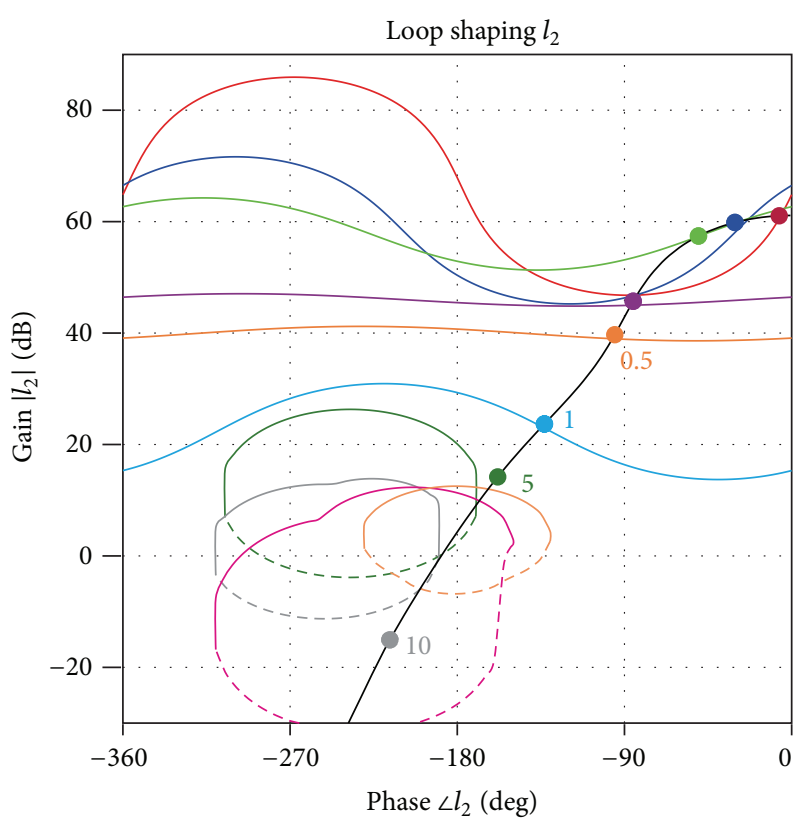

(b)

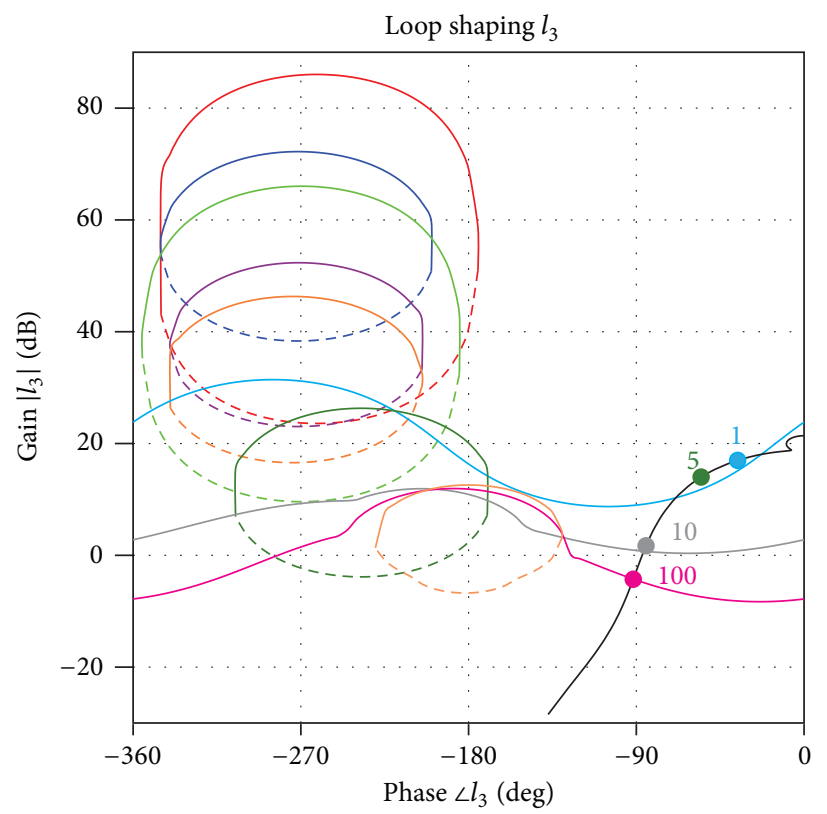

(c)

FIGURE 13: Loop-shaping of three parallel controllers.

$$
\begin{gathered}
c_{2}=\frac{32.4(s+0.1)}{(s+0.0087)(s+0.63)(s+5.27)}, \\
c_{3}=\frac{6.155(s+0.01)}{(s+0.007355)(s+0.7128)}
\end{gathered}
$$

Figure 14 depicts the expected feedback sharing along the frequency band (Table 3) in terms of branch open-loop functions $\left|l_{i}\right|$ and their contribution to the global open-loop function $\left|l_{t}\right|=\left|l_{1}+l_{2}+l_{3}\right|$ in $\mathrm{dB}$ units.

The originality of this theoretical example relapses mainly in two challenges: facing regulatory problems with more than two manipulated inputs and dealing with parallel plants when some of them must work in disjoint bands of frequencies. Note that $p_{1}$ joins first at $\omega \in[0.001,0.01]$ and later on at $\omega \in[0.5,10]$. This causes that there is not an equivalent for $c_{1}$ (22), $c_{2}$ (23), and $c_{3}$ (24) in a serial structure (Figure $1(\mathrm{a}))$. Let us rename serial controllers as $c_{1}^{s}, c_{2}^{s}$, and $c_{3}^{s}$. The equivalents can be computed as $c_{1}^{s}=c_{1}$, and $c_{2}^{s}=-c_{2} / c_{1}, c_{3}^{s}=$ $-c_{3} / c_{2}$, which would yield a nonproper controller $c_{3}^{s}$. On the other hand, it is not feasible either a straight away design of the serial controllers $c_{i}^{s}$ using a main-vernier strategy, since the load sharing planning (Table 3 ) does not allow sorting the 


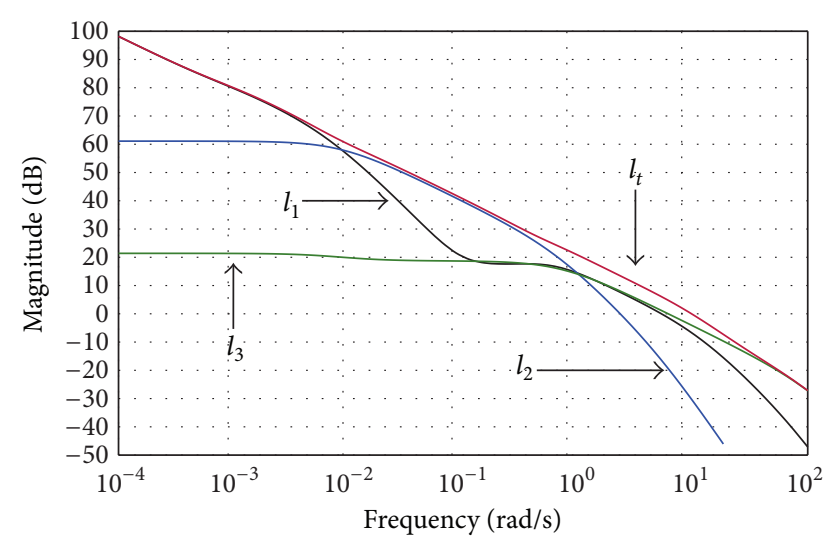

FIgURE 14: Open-loop responses on magnitude Bode diagram.

plants from faster to slower ( $p_{1}$ joins at discontinuous intervals of frequencies). That is, the serial strategy is conceived for that each plant works in one and only one frequency interval.

Remark 5. This example tries to illustrate the new methodology ability (i) to solve regulatory problems of more than two manipulated inputs, which are frequent in engineering applications but are not so common in the scientific literature and (ii) to deal with parallel plants when some of them must work in disjoint bands of frequencies. For this last case, a serial structure of controllers (main-vernier) fails.

\section{Conclusions}

In attention to engineering application demands, a parallel structure of controllers has been proposed to solve MISO regulatory problems, which concern robust stability and robust disturbance rejection. MISO plant models included parametric uncertainty. Inside a QFT framework, a new methodology of design was proposed with the aim of tuning a set of controllers that met the robust specifications, and simultaneously, each controller used the least possible gain at each frequency. The keypoint was to quantify the feedback demanded by each plant at each frequency to meet the specifications by itself. This allowed a planning of the best load sharing along the frequency band. At each frequency, either one or several plants in collaboration must assume feedback responsibilities to meet the specifications or the plant/s participation must be inhibited for feedback saving. To accomplish it, a sequential design of the parallel controllers was fully detailed, using current QFT software tools for bound computations and for loop-shapings.

The abilities and engineering interest of the new methodology were demonstrated through several examples. The first one illustrated the methodology and detailed the benefits of a parallel structure of robust controllers in comparison with a single robust master controller. The main advantages are (i) to reduce the sensor noise amplification in the actuators of the slowest plants, which are conceived to work in the band of lower frequencies and provide the stationary and (ii) to avoid unnecessary offsets in the actuators of the faster plants, and consequently, that their full range of actuation is available to speed up the response with less risk of saturation. Nevertheless, these are also the aims of current MISO methodologies, whose weak points have been also overcome by the new technique. In particular, a second engineering example exhibited the difficulties of tuning a parallel control using the master-slave philosophy. The challenge is mainly the filter (slaves) design due to plant uncertainties and the dynamic incorporation of disturbances, which are common in reallife plants. A third example illustrated the weak points of a chained serial structure of controllers (main-vernier), which fails when plants must work in disjoint bands of frequencies. In any case, the proposed parallel structure can always be converted to both master-slaves or main-vernier structures. The inverse procedure was demonstrated as not always being possible. In addition, the third example showed the difficulty of facing a MISO problem with three manipulabled variables, which are not common in the scientific literature but are frequent in engineering applications.

\section{Conflict of Interests}

The authors declare that there is no conflict of interests regarding the publication of this paper.

\section{Acknowledgment}

The authors are grateful for the assistance provided by La Rioja Government through Project IMPULSA 2010/01 and through ADER Grant 2012-I-IDD-00093.

\section{References}

[1] M. N. Marwali, J.-W. Jung, and A. Keyhani, "Control of distributed generation systems-part II: load sharing control," IEEE Transactions on Power Electronics, vol. 19, no. 6, pp. 15511561, 2004.

[2] E. Eitelberg, "Macrodynamic feedback interaction between trade and production," International Journal of Robust and Nonlinear Control, vol. 17, no. 2-3, pp. 203-224, 2007.

[3] M. Garcia-Sanz and F. Y. Hadaegh, "Load-sharing robust control of spacecraft formations: deep space and low Earth elliptic orbits," IET Control Theory and Applications, vol. 1, no. 2, pp. 475-484, 2007.

[4] I. Horowitz, Synthesis of Feedback Systems, 1963.

[5] M. Kano and M. Ogawa, "The state of the art in chemical process control in Japan: good practice and questionnaire survey," Journal of Process Control, vol. 20, no. 9, pp. 969-982, 2010.

[6] S. K. Jha and N. Kaistha, "Valve positioning control for process through-put maximization," Chemical Engineering Research and Design, vol. 85, no. 11, pp. 1465-1475, 2007.

[7] B. J. Allison and A. J. Isaksson, "Design and performance of mid-ranging controllers," Journal of Process Control, vol. 8, no. 5-6, pp. 469-474, 1998.

[8] B. J. Allison and S. Ogawa, "Design and tuning of valve position controllers with industrial applications," Transactions of the Institute of Measurement and Control, vol. 25, no. 1, pp. 3-16, 2003. 
[9] C.-C. Yu and W. L. Luyben, "Analysis of valve-position control for dual-input processes," Industrial and Engineering Chemistry Fundamentals, vol. 25, no. 3, pp. 344-350, 1986.

[10] R. B. McLain, M. J. Kurtz, M. A. Henson, and F. J. Doyle III, "Habituating control for nonsquare nonlinear processes," Industrial and Engineering Chemistry Research, vol. 35, no. 11, pp. 4067-4077, 1996.

[11] R. Monroy-Loperena, R. Solar, and J. Alvarez-Ramirez, "Balanced control scheme for reactor/separator processes with material recycle," Industrial and Engineering Chemistry Research, vol. 43, no. 8, pp. 1853-1862, 2004.

[12] A. Velasco-Perez, J. Alvarez-Ramirez, A. Velasco, and R. SolarGonzalez, "Control múltiple entrada una salida (miso) de un cstr," Revista Mexicana De Ingeniería Química, vol. 10, no. 2, pp. 321-331, 2011.

[13] O. A. Prado-Rubio, S. B. Jorgensen, and G. Jonsson, "Ph control structure design for a periodically operated membrane separation process," Computers and Chemical Engineering, vol. 43, pp. 120-129, 2012.

[14] S. Haugwitz, P. Hagander, and T. Norén, "Modeling and control of a novel heat exchange reactor, the open plate reactor," Control Engineering Practice, vol. 15, no. 7, pp. 779-792, 2007.

[15] S. Velut, L. de Maré, and P. Hagander, "Bioreactor control using a probing feeding strategy and mid-ranging control," Control Engineering Practice, vol. 15, no. 2, pp. 135-147, 2007.

[16] M. Karlsson, O. Slätteke, B. Wittenmark, and S. Stenström, "Reducing moisture transients in the paper-machine drying section with the mid-ranging control technique," Nordic Pulp and Paper Research Journal, vol. 20, no. 2, pp. 150-155, 2005.

[17] A. Velasco-Pérez and J. Alvarez-Ramírez, "Algorithm for parallel control for an aerobic reactor with a recirculation current," Revista Mexicana De Ingeniería Química, vol. 6, no. 2, pp. 229236, 2007.

[18] M. A. Henson, B. A. Ogunnaike, and J. S. Schwaber, "Habituating control strategies for process control," AIChE Journal, vol. 41, no. 3, pp. 604-618, 1995.

[19] D. F. Marselle, M. Morari, and D. F. Rudd, "Design of resilient processing plants-II design and control of energy management systems," Chemical Engineering Science, vol. 37, no. 2, pp. 259270, 1982.

[20] L. Giovanini, “Cooperative-feedback control," ISA Transactions, vol. 46, no. 3, pp. 289-302, 2007.

[21] G. F. Fauth and F. G. Shinskey, "Advanced control of distillation columns," Chemical Engineering Progress, vol. 71, no. 6, pp. 4954, 1975.

[22] F. G. Shinskey, "Energy-conserving control systems for distillation units," Chemical Engineering Progress, vol. 72, no. 5, pp. 73-78, 1976.

[23] F. G. Shinskey, "Control systems can save energy," Chemical Engineering Progress, vol. 74, no. 5, pp. 43-46, 1978.

[24] S. J. Williams, D. Hrovat, C. Davey, D. Maclay, J. W. V. Crevel, and L. F. Chen, "Idle speed control design using an H-infinity approach," in Proceedings of the American Control Conference, pp. 1950-1956, June 1989.

[25] S. Jayasuriya and M. A. Franchek, "A qft-type design methodology for a parallel plant structure and its application in idle speed control," International Journal of Control, vol. 60, no. 5, pp. 653670, 1994.
[26] V. Besson and A. T. Shenton, "Interactive parameter space design for robust performance of MISO control systems," IEEE Transactions on Automatic Control, vol. 45, no. 10, pp. 1917-1924, 2000.

[27] P. Gorzelic, E. Hellstrom, A. Stefanopoulou, L. Jiang, and S. Gopinath, "A coordinated approach for throttle and wastegate control in turbocharged spark ignition engines," in Proceedings of the 24th Control and Decision Conference (CCDC '12), pp. 1524-1529, 2012.

[28] N. Ravi, H.-H. Liao, A. F. Jungkunz, and J. C. Gerdes, "Midranging control of a multi-cylinder HCCI engine using split fuel injection and valve timings," in Proceedings of the 6th IFAC Symposium Advances in Automotive Control (AAC '10), pp. 797802, July 2010.

[29] S. Jade, E. Hellstrom, L. Jiang, and A. G. Stefanopoulou, "Fuel governor augmented control of recompression hcci combustion during large load transients," in Proceedings of the American Control Conference, pp. 2084-2089, 2012.

[30] S. Jade, J. Larimore, E. Hellstrom, L. Jiang, and A. G. Stefanopoulou, "Enabling large load transitions on multicylinder recompression hcci engines using fuel governors," in Proceedings of the American Control Conference, pp. 4423-4428, 2013.

[31] J. Zhai, Y. Huang, S. Schroeck, W. Messner, D. D. Stancil, and T. E. Schlesinger, "High bandwidth electro-optic scanner for optical data storage," Japanese Journal of Applied Physics, vol. 39, no. 2, pp. 883-887, 2000.

[32] A. Al Mamun, I. Mareels, T. H. Lee, and A. Tay, "Dual stage actuator control in hard disk drive-a review," in Proceedings of the 29th Annual Conference of the IEEE Industrial Electronics Society, pp. 2132-2137, November 2003.

[33] T. Gao, C. Du, C. P. Tan, Z. He, J. Yang, and L. Xie, "High bandwidth control design and implementation for a dualstage actuation system with a microthermal actuator," IEEE Transactions on Magnetics, vol. 49, no. 3, pp. 1082-1087, 2013.

[34] J. Sun, F. Cameron, and B. W. Bequette, "A habituating blood glucose control strategy for the critically ill," Journal of Process Control, vol. 22, no. 8, pp. 1411-1421.

[35] K. Aström and T. Hägglund, Control Pid Avanzado, Pearson Prentice Hall, 2009.

[36] V. Lersbamrungsuk, T. Srinophakun, S. Narasimhan, and S. Skogestad, "Control structure design for optimal operation of heat exchanger networks," AIChE Journal, vol. 54, no. 1, pp. 150$162,2008$.

[37] J. Alvarez-Ramirez and H. Puebla, "On classical PI control of chemical reactors," Chemical Engineering Science, vol. 56, no. 6, pp. 2111-2121, 2001.

[38] J. Alvarez-Ramirez, A. Velasco, and G. Fernandez-Anaya, "A note on the stability of habituating process control," Journal of Process Control, vol. 14, no. 8, pp. 939-945, 2004.

[39] B. J. Lurie and P. Enright, Classical Feedback Control: With MATLAB, Control Engineering, Taylor \& Francis, 2000.

[40] R. L. Grogan, G. H. Blackwood, and R. J. Calvet, "Optical delay line nanometer level pathlength control law design for spacebased interferometry," in Proceedings of the International Society for Optical Engineering (SPIE '98), pp. 14-25, March 1998.

[41] G. W. Neat and A. Abramovici, "Control technology lessons learned: case study using the micro-precision interferometer testbed," in Proceedings of the American Control Conference, vol. 2,1998 . 
[42] J. Daeges, B. Lurie, and A. Bhanji, "An improved high-voltage dc regulator for a radar and communication transmitter," in Proceedings of the 18th IEEE Conference Record of the Power Modulator Symposium, pp. 106-108, 1988.

[43] Q.-G. Wang, Y. Zhang, W.-J. Cai, Q. Bi, and C.-C. Hang, "Cooperative control of multi-input single-output processes: online strategy for releasing input saturation," Control Engineering Practice, vol. 9, no. 5, pp. 491-500, 2001.

[44] L. L. Giovanini, "Flexible-structure control: a strategy for releasing input constraints," ISA Transactions, vol. 43, no. 3, pp. 361-376, 2004.

[45] E. Eitelberg, Load Sharing Control, NOYB Press, 1999.

[46] E. Eitelberg, "Load sharing in a multivariable temperature control system," Control Engineering Practice, vol. 7, no. 11, pp. 1369-1377, 1999.

[47] E. Eitelberg, "Some peculiarities of load sharing control," International Journal of Robust and Nonlinear Control, vol. 13, no. 7, pp. 607-618, 2003.

[48] S. J. Schroeck, W. C. Messner, and R. J. McNab, "On compensator design for linear time-invariant dual-input single-output systems," IEEE/ASME Transactions on Mechatronics, vol. 6, no. 1, pp. 50-57, 2001.

[49] S. Brennan and A. Alleyne, "Integrated vehicle control via coordinated steering and wheel torque inputs," in Proceedings of the American Control Conference, pp. 7-12, June 2001.

[50] B. J. Allison and J. B. Ball, "Constrained model predictive control of blow tank consistency," Control Engineering Practice, vol. 12, no. 7, pp. 837-845, 2004.

[51] S. Haugwitz, M. Karlsson, S. Velut, and P. Hagander, "Antiwindup in mid-ranging control," in Proceedings of the 44th IEEE Conference on Decision and Control, and the European Control Conference (CDC-ECC '05), pp. 7570-7575, December 2005.

[52] M. Karlsson, O. Slatteke, T. Hagglund, and S. Stenstrom, "Feedforward control in the paper machine drying section," in Proceedings of the American Control Conference, 2006.

[53] S. Gayadeen and W. Heath, "An internal model control approach to mid-ranging control," in Proceedings of the 7th IFAC International Symposium on Advanced Control of Chemical Processes (ADCHEM '09), pp. 542-547, July 2009.

[54] W. P. Heath and S. Gayadeen, "Simple robustness measures for control of miso and simo plants," in IFAC Proceedings, vol. 18, pp. 11356-11361, 2011.

[55] I. Horowitz, Quantitative Feedback Design Theory (QFT), QFT Publications, Boulder, Colorado, USA, 1993.

[56] P.-O. Gutman, E. Horesh, R. Guetta, and M. Borshchevsky, "Control of the aero-electric power station-an exciting qft application for the 21st century," International Journal of Robust and Nonlinear Control, vol. 13, no. 7, pp. 619-636, 2003.

[57] F. G. Shinskey and J. H. P. Castellanos, "Sistemas de control de procesos: aplicación, diseño y sintonización," in Sistemas De Control De Procesos: Aplicación, Diseño Y Sintonización, McGraw-Hill, 1996.

[58] A. Gera and I. Horowitz, "Optimization of the loop transfer function," International Journal of Control, vol. 31, no. 2, pp. 389398,1980

[59] C. Borghesani, Y. Chait, and O. Yaniv, Quantitative Feedback Theory Toolbox. For Use With Matlab, Terasoft, 2nd edition, 2002.
[60] F. Instruments, Limited Proces control, http://www.fbk.com.

[61] J. Rico and M. Gil-Martinez, "Multivariable QFT robust control of a heat exchanger," in Proceedings of the 19th Mediterranean Conference on Control and Automation (MED '11), pp. 588-593, June 2011. 


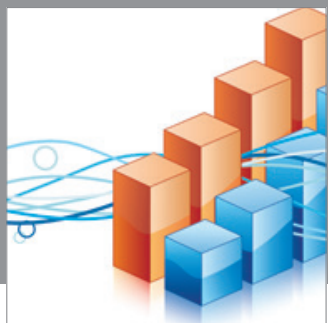

Advances in

Operations Research

mansans

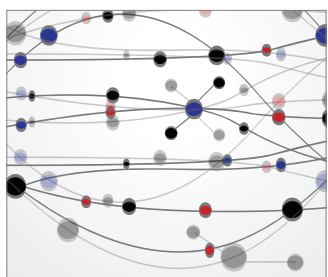

The Scientific World Journal
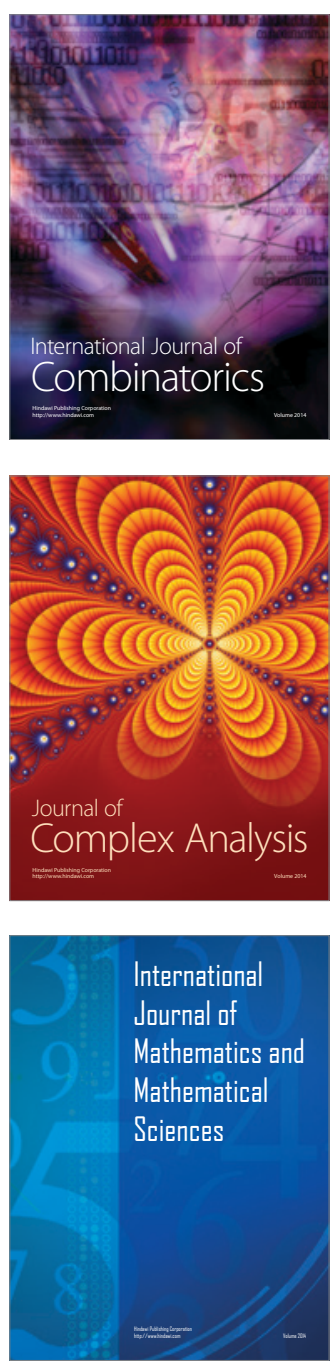
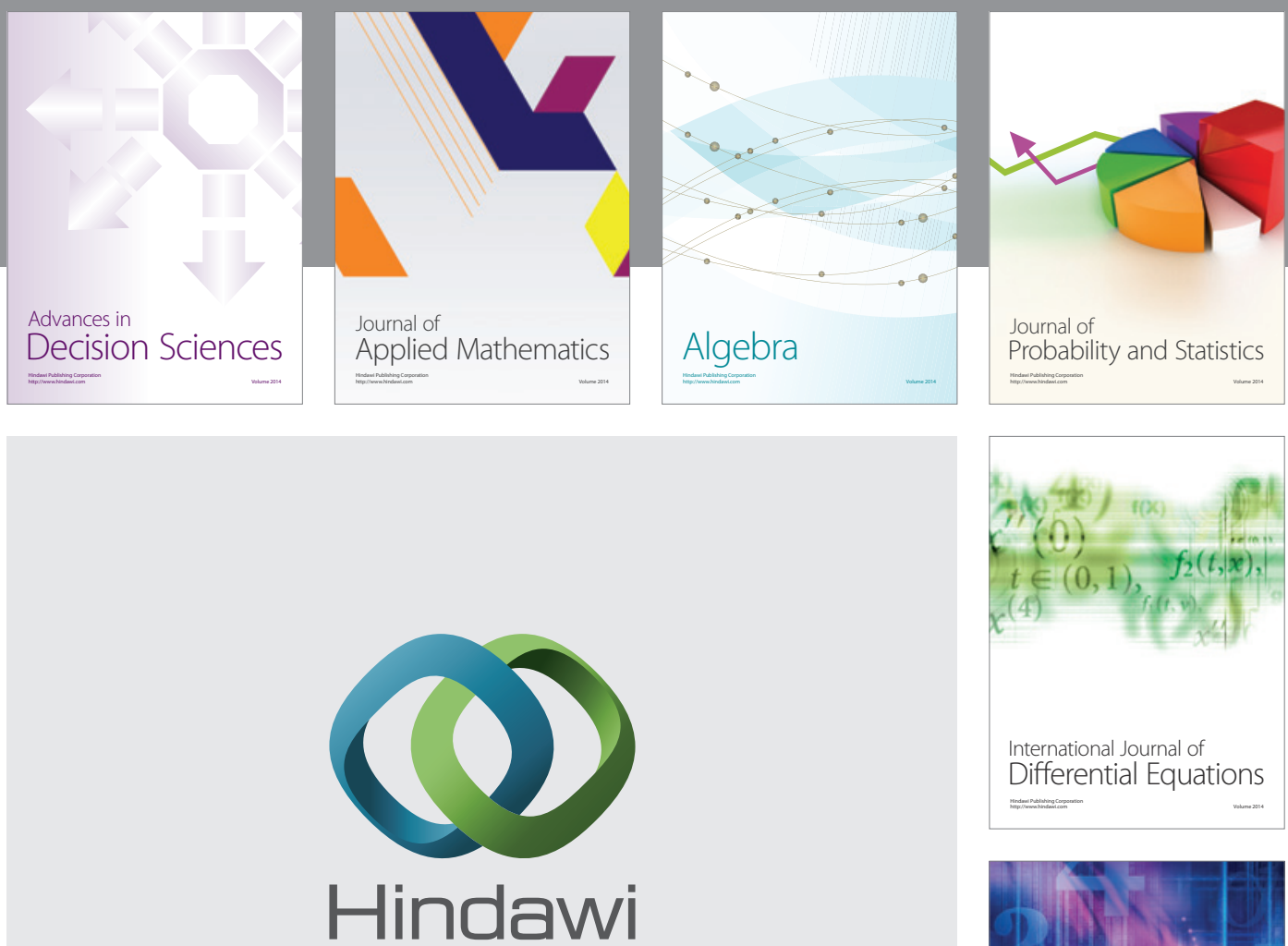

Submit your manuscripts at http://www.hindawi.com
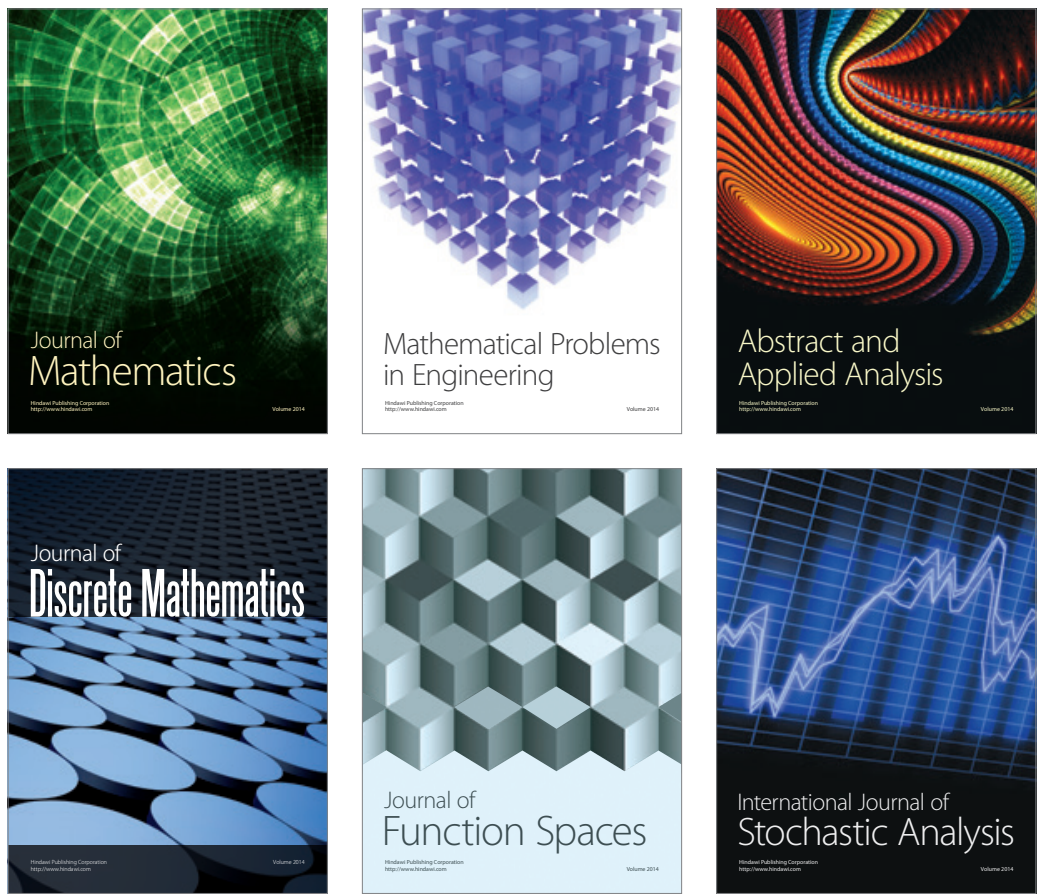

Journal of

Function Spaces

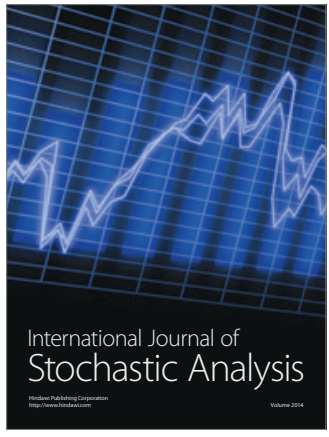

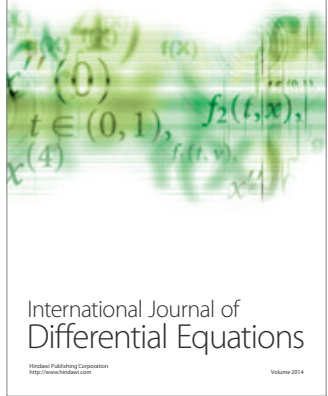
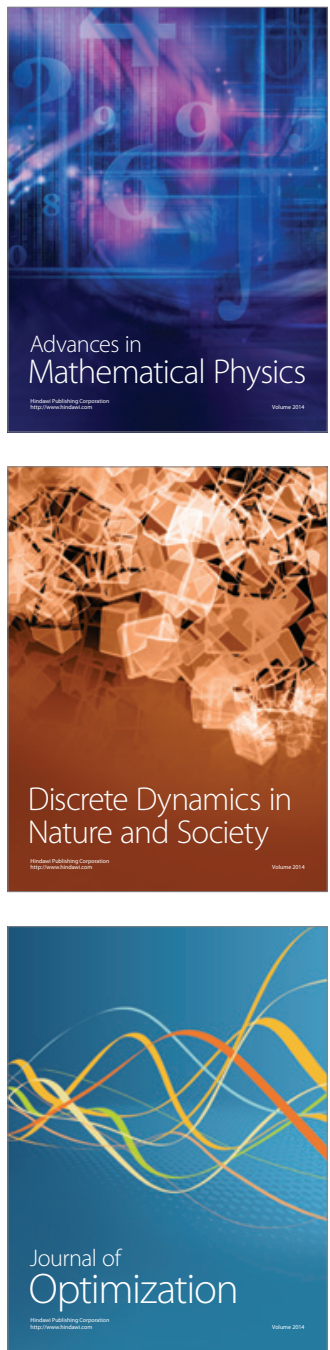